\title{
Inference for types and structured families of commutative orthogonal block structures
}

\section{Francisco Carvalho, João T. Mexia, Carla Santos \& Célia Nunes}

\section{Metrika}

International Journal for Theoretical and Applied Statistics

\section{ISSN 0026-1335}

Volume 78

Number 3

Metrika (2015) 78:337-372

DOI 10.1007/s00184-014-0506-8

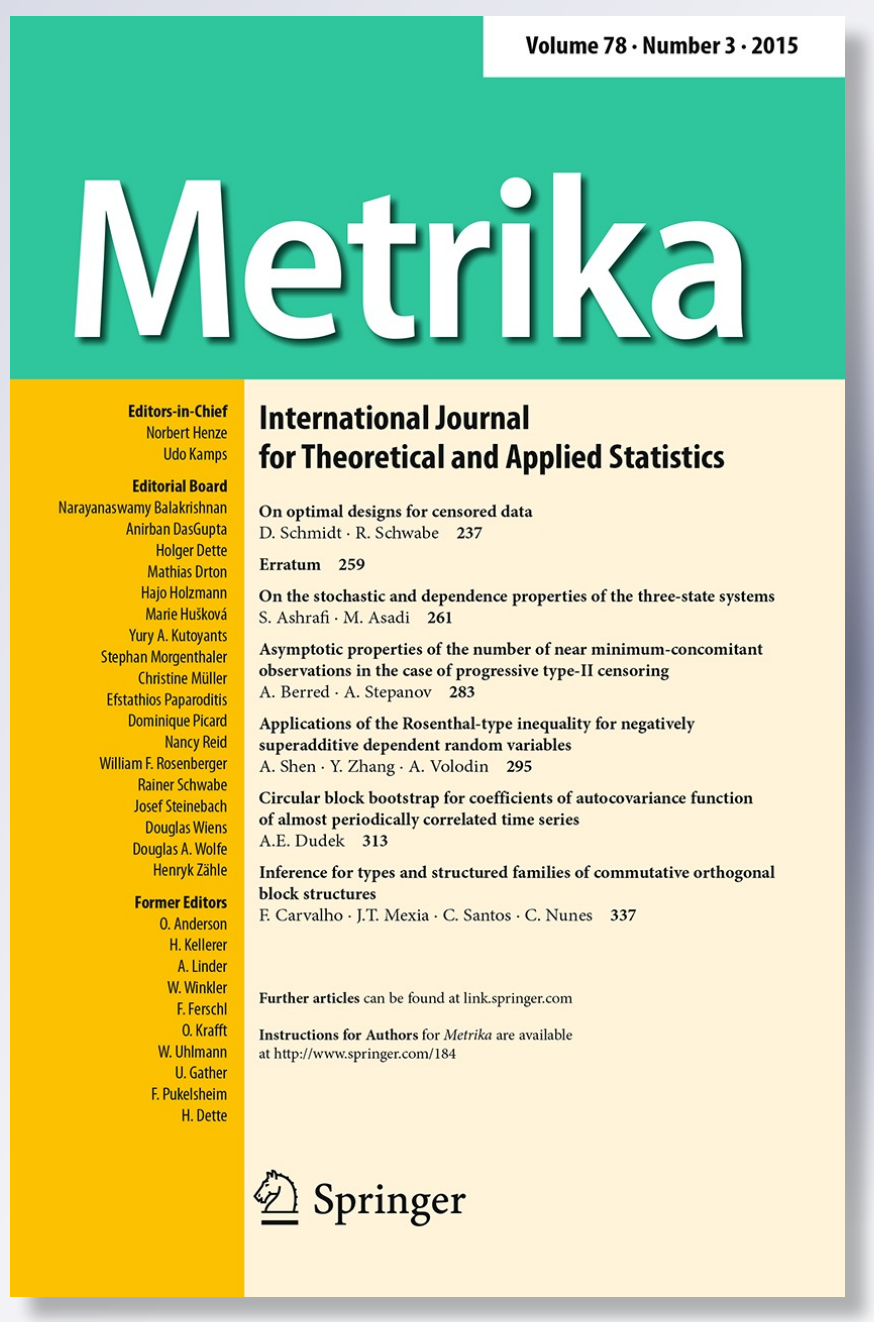

Springer 
Your article is protected by copyright and all rights are held exclusively by SpringerVerlag Berlin Heidelberg. This e-offprint is for personal use only and shall not be selfarchived in electronic repositories. If you wish to self-archive your article, please use the accepted manuscript version for posting on your own website. You may further deposit the accepted manuscript version in any repository, provided it is only made publicly available 12 months after official publication or later and provided acknowledgement is given to the original source of publication and a link is inserted to the published article on Springer's website. The link must be accompanied by the following text: "The final publication is available at link.springer.com". 


\title{
Inference for types and structured families of commutative orthogonal block structures
}

\author{
Francisco Carvalho • João T. Mexia • \\ Carla Santos - Célia Nunes
}

Received: 8 February 2013 / Published online: 19 August 2014

(C) Springer-Verlag Berlin Heidelberg 2014

\begin{abstract}
Models with commutative orthogonal block structure, COBS, have orthogonal block structure, OBS, and their least square estimators for estimable vectors are, as it will be shown, best linear unbiased estimator, BLUE. Commutative Jordan algebras will be used to study the algebraic structure of the models and to define special types of models for which explicit expressions for the estimation of variance components are obtained. Once normality is assumed, inference using pivot variables is quite straightforward. To illustrate this class of models we will present unbalanced examples before considering families of models. When the models in a family correspond to the
\end{abstract}

\footnotetext{
F. Carvalho $\cdot$ J. T. Mexia

CMA - Centro de Matemática e Aplicações, Faculdade de Ciências e Tecnologia, Universidade Nova de Lisboa, 2829-516 Caparica, Portugal

F. Carvalho

Unidade Departamental de Matemática e Física, Instituto Politécnico de Tomar, 2300-313 Tomar, Portugal

J. T. Mexia

Departamento de Matemática, Faculdade de Ciências e Tecnologia, Universidade Nova de Lisboa, 2829-516 Caparica, Portugal

C. Santos

Departamento de Matemática e Ciências Físicas, Escola Superior de Tecnologia e Gestão, Instituto Politécnico de Beja, 7800-295 Beja, Portugal

C. Nunes $(\varangle)$

Departamento de Matemática, Faculdade de Ciências, Universidade da Beira Interior, 6201-001 Covilhã, Portugal

e-mail: celian@ubi.pt

C. Nunes

Centro de Matemática, Universidade da Beira Interior, 6201-001 Covilhã, Portugal
} 
treatments of a base design, the family is structured. It will be shown how, under quite general conditions, the action of the factors in the base design on estimable vectors, can be studied.

Keywords Commutative orthogonal block structure - Commutative Jordan algebras $\cdot$ Estimation $\cdot$ Mixed linear models

\section{Introduction}

Models with orthogonal block structure, OBS, introduced by Nelder (1965a, b) continue to play an important part in the theory of randomized block designs, see Caliński and Kageyama (2000, 2003).

The family $\mathscr{V}$ of variance-covariance matrices of those models is constituted by the

$$
\mathscr{V}=\left\{\sum_{j=1}^{m^{\circ}} \gamma_{j}^{\circ} \boldsymbol{Q}_{j}^{\circ}\right\}
$$

where the $\gamma_{1}^{\circ}, \ldots, \gamma_{m}^{\circ}$ are unknown non negative constants, and the $\boldsymbol{Q}_{1}^{\circ}, \ldots, \boldsymbol{Q}_{m}^{\circ}$ are known mutually orthogonal orthogonal projection matrices, such that

$$
\sum_{j=1}^{m^{\circ}} \boldsymbol{Q}_{j}^{\circ}=\boldsymbol{I}_{n} .
$$

When the matrices $\boldsymbol{Q}_{1}^{\circ}, \ldots, \boldsymbol{Q}_{m}^{\circ}$ commute with the orthogonal projection matrix $\boldsymbol{T}$ on the space spanned by the mean vector of the model, it has commutative orthogonal block structure, COBS, and the least square estimators of estimable vectors are best linear unbiased estimator, BLUE, see Zmyślony (1980). ${ }^{1}$

In carrying out the estimation for models with COBS, we use commutative Jordan algebras of symmetric matrices, CJAS, in expressing the algebraic structure of those models. Namely, see Fonseca et al. (2003), the uniformly minimum variance unbiased estimator, UMVUE, for variance components, in normal models with balanced cross nesting, can be represented as linear combinations of mean squares.

For instance, in a random effects model in Khuri et al. (1998), in which a first factor crosses with a second one that nests a third one, the estimation of variance components for the second factor is a linear combination of four mean squares. Two of these mean squares have positive coefficients and the two others have negative coefficients. Thus we cannot use the usual $\mathrm{F}$ test for testing the nullity of their variance components, and we may have to use a generalized F test, see Michalski and Zmyślony (1996).

In this work after considering single models we will study structured families. A first example of such families is that of multiregression designs, see Mexia (1987). Then for each treatment of a base design we have a linear regression on the same

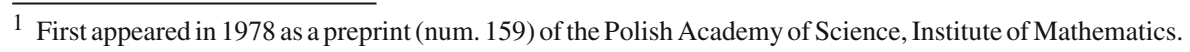


variables. The matrices of values of controlled variables and the variance of the error are assumed to be the same for the different regressions. The inference for this family of regressions is centered on the vectors of coefficients or, more generally, on estimable vectors, leading to interesting results, see Moreira et al. (2005a, b), Moreira and Mexia (2007) and Moreira (2008).

In the structured families we will consider, regressions are replaced by COBS but the inference will still be centered on estimable vectors. Both the common structure of the models in the family and the structure of the base design will play their part in the analysis of structured families of COBS.

In the next section we will cover the basic framework of this work. Next we consider inference for single COBS and, then, the joint analysis of structured families. Finally we make some concluding remarks.

Throughout the paper we will consider a two factor model to illustrate our results. To avoid confusion the references to that model will be separated from the main text. This illustration will easily demonstrate the advantage of the technique presented for computational use.

\section{Algebras and models}

CJAS are linear spaces constituted by symmetric matrices that commute and contain the square of their matrices. These structures where introduced by Jordan et al. (1934) in a new approach to Quantum Mechanics. Later on they were used by Seely (1970a, b, 1971), Seely and Zyskind (1971), that named them as quadratic spaces, and used them to perform linear statistical inference. This approach had many interesting developments, see e. g. Zmyślony and Drygas (1992), VanLeeuwen et al. (1998, 1999), and Fonseca et al. (2006, 2007, 2008). This paper follows this trend.

Every CJAS, $\mathscr{A}$, has an unique basis constituted by mutually orthogonal orthogonal projection matrices, see Seely (1971) and Seely and Zyskind (1971). This basis will be the principal basis of $\mathscr{A}, p b(\mathscr{A})$. Due to the existence of the principal basis of $\mathscr{A}$, we can see that every CJAS, $\mathscr{A}$, contains the product of its matrices and that every orthogonal projection matrix belonging to $\mathscr{A}$ is the sum of matrices in $p b(\mathscr{A})$. The rank of the orthogonal projection matrix being the sum of the ranks of these matrices of the $p b(\mathscr{A})$, so if the orthogonal projection matrix has rank one it has to belong to the principal basis. For instance if $\frac{1}{n} \boldsymbol{J}_{n} \in \mathscr{A}$, with $\boldsymbol{J}_{n}=\mathbf{1}_{n} \mathbf{1}_{n}^{\top}$ and $\mathbf{1}_{n}$ denoting the vector with all $n$ components equal to 1 , we take $\boldsymbol{Q}_{1}=\frac{1}{n} \boldsymbol{J}_{n}$ and say that $\mathscr{A}$ is regular.

Given the principal basis of $\mathscr{A}, \mathscr{Q}=\left\{\boldsymbol{Q}_{1}, \ldots, \boldsymbol{Q}_{m}\right\}=p b(\mathscr{A})$ and $\boldsymbol{W} \in \mathscr{A}$, we have $\boldsymbol{W}=\sum_{j=1}^{m} a_{j} \boldsymbol{Q}_{j}=\sum_{j \in \mathscr{C}} a_{j} \boldsymbol{Q}_{j}$, with $\mathscr{C}=\left\{j: a_{j} \neq 0\right\}$. The MoorePenrose inverse of $\boldsymbol{W}$ is given by $\boldsymbol{W}^{\dagger}=\sum_{j \in \mathscr{C}} a_{j}^{-1} \boldsymbol{Q}_{j}$ and $\boldsymbol{W} \boldsymbol{W}^{\dagger}=\sum_{j \in \mathscr{C}} \boldsymbol{Q}_{j}$, with $\boldsymbol{W} \boldsymbol{W}^{\dagger}$ the orthogonal projection matrix on the range space of $\boldsymbol{W}, R(\boldsymbol{W})$. So, $\mathscr{A}$ will contain the orthogonal projection matrix on the range spaces of its matrices and their Moore-Penrose inverses.

Moreover it will contain invertible matrices, if and only if, $\sum_{j=1}^{m} \boldsymbol{Q}_{j}=\boldsymbol{I}_{n}$ and, then $\boldsymbol{W} \in \mathscr{A}$ is regular, when and only when $\mathscr{C}=\{1, \ldots, m\}$. We will say that such CJAS are complete. 
A family $\mathscr{M}=\left\{\boldsymbol{M}_{1}, \ldots, \boldsymbol{M}_{w}\right\}$ of symmetric matrices is commutative if its matrices commute. Now $\mathscr{M}$ is commutative if and only if its matrices are diagonalized by the same orthogonal matrix $\boldsymbol{P}$, see Schott (1997). We will then have $\mathscr{M} \subseteq \mathscr{V}(\boldsymbol{P})$, with $\mathscr{V}(\boldsymbol{P})$ the family of matrices diagonalized by $\boldsymbol{P}$. Since $\mathscr{V}(\boldsymbol{P})$ is a CJAS, $\mathscr{M}$ is contained in a CJAS, if and only if, it is commutative. Given the interception of CJAS being a CJAS, so if $\mathscr{M}$ is commutative, there will be a minimum CJAS, $\mathscr{A}(\mathscr{M})$, that contains $\mathscr{M}$. We will say that this CJAS is generated by $\mathscr{M}$. Namely, if the matrices of $\mathscr{Q}$ are mutually orthogonal orthogonal projection matrices, we will have $\mathscr{Q}=p b(\mathscr{A}(\mathscr{Q}))$.

Since the matrices in $\mathscr{M}$ commute, the linear space $\mathscr{L}(\mathscr{M})$ constituted by all linear combinations of the matrix products $\boldsymbol{M}_{1}^{p_{1}} \ldots \boldsymbol{M}_{w}^{p_{w}}$, for all choices of non negative integers $p_{1}, \ldots, p_{w}$, is commutative and contains the square of its matrices, so it will be a CJAS. Moreover, $\mathscr{L}(\mathscr{M})$ is contained in any CJAS that contains $\mathscr{A}(\mathscr{M})$ and we have

$$
\mathscr{L}(\mathscr{M})=\mathscr{A}(\mathscr{M})
$$

Thus, if $\boldsymbol{W}$ is a symmetric matrix that commutes with the matrices of $\mathscr{M}$, it will commute with those of $\mathscr{A}(\mathscr{M})$ and so with those in the principal basis $\mathscr{Q}$ of $\mathscr{A}(\mathscr{M})$. Inversely, if $\boldsymbol{W}$ commutes with the matrices in $\mathscr{Q}$, it will commute with those in $\mathscr{A}(\mathscr{M})$ and so with those in $\mathscr{M}$.

We have established

Lemma 1 A symmetric matrix commutes with the matrices in $\mathscr{M}$ if and only if it commutes with the matrices in $\mathscr{Q}=p b(\mathscr{A}(\mathscr{M}))$.

Given the known matrices $\boldsymbol{X}_{i}, i=0, \ldots, w$, the mixed model

$$
\boldsymbol{y}=\sum_{i=0}^{w} \boldsymbol{X}_{i} \boldsymbol{\beta}_{i}
$$

with $\boldsymbol{\beta}_{0}$ fixed and the $\boldsymbol{\beta}_{1}, \ldots, \boldsymbol{\beta}_{w}$ random, independent, with null mean vectors and variance-covariance matrices $\sigma_{i}^{2} \boldsymbol{I}_{c_{i}}, i=1, \ldots, w$, where $c_{i}, i=1, \ldots, w$, are the number of components of $\boldsymbol{\beta}_{i}, i=1, \ldots, w$, will have mean vector and variancecovariance matrix

$$
\left\{\begin{array}{l}
\boldsymbol{\mu}=\boldsymbol{X}_{0} \boldsymbol{\beta}_{0} \\
\boldsymbol{V}=\sum_{i=1}^{w} \sigma_{i}^{2} \boldsymbol{M}_{i}
\end{array}\right.
$$

where $\boldsymbol{M}_{i}=\boldsymbol{X}_{i} \boldsymbol{X}_{i}^{\top}, i=1, \ldots, w$. Thus the family of the variance-covariance matrices will be

$$
\mathscr{V}=\left\{\sum_{i=1}^{w} \sigma_{i}^{2} \boldsymbol{M}_{i} ; \quad \sigma_{i}^{2} \geq 0, \quad i=1, \ldots, w\right\}
$$

When the matrices $\boldsymbol{M}_{1}, \ldots, \boldsymbol{M}_{w}$ are linearly independent, $\sum_{i=1}^{w} \sigma_{1, i}^{2} \boldsymbol{M}_{i}=$ $\sum_{i=1}^{w} \sigma_{2, i}^{2} \boldsymbol{M}_{i}$ implies $\sigma_{1, i}^{2}=\sigma_{2, i}^{2}, i=1, \ldots, w$, and the variance components identify $\boldsymbol{V}$. 
We point out the possibility of having singular variance-covariance matrix.

If the column vectors of $\boldsymbol{X}_{0}$ are linearly independent, we have

$$
\boldsymbol{\beta}_{0}=\boldsymbol{X}_{0}^{\dagger} \boldsymbol{\mu}
$$

with $\boldsymbol{X}_{0}^{\dagger}=\left(\boldsymbol{X}_{0}^{\top} \boldsymbol{X}_{0}\right)^{-1} \boldsymbol{X}_{0}^{\top}$, and $\boldsymbol{T}=\boldsymbol{X}_{0}\left(\boldsymbol{X}_{0}^{\top} \boldsymbol{X}_{0}\right)^{-1} \boldsymbol{X}_{0}^{\top}$, so

$$
\widetilde{\boldsymbol{\beta}}_{0}=\boldsymbol{X}_{0}^{\dagger} \boldsymbol{y}
$$

will be the least squares estimator, LSE, of $\boldsymbol{\beta}_{0}$, see e.g. Kariya and Kurata (2004).

$\nabla$

For instance, if we have a fixed effect factor with $a$ levels that crosses with a random effects factor with $b$ levels, we have

$$
\boldsymbol{y}=\sum_{i=0}^{2} \boldsymbol{X}_{i} \boldsymbol{\beta}_{i},
$$

with

$$
\left\{\begin{array}{l}
\boldsymbol{X}_{0}=\boldsymbol{I}_{a} \otimes \mathbf{1}_{b} \\
\boldsymbol{X}_{1}=\mathbf{1}_{a} \otimes \boldsymbol{I}_{b} \\
\boldsymbol{X}_{2}=\boldsymbol{I}_{a} \otimes \boldsymbol{I}_{b}
\end{array}\right.
$$

where $\otimes$ denotes the Kronecker matrix product and $\boldsymbol{\beta}_{0}\left[\boldsymbol{\beta}_{1} ; \boldsymbol{\beta}_{2}\right]$ the vector of effects for the levels of the first factor [effects for the levels of the second factor; interactions between levels of the two factors]. While $\boldsymbol{\beta}_{0}$ will be fixed, $\boldsymbol{\beta}_{1}$ and $\boldsymbol{\beta}_{2}$ will have null mean vectors and variance-covariance matrices $\sigma_{1}^{2} \boldsymbol{I}_{b}$ and $\sigma_{2}^{2} \boldsymbol{I}_{n}$, with $n=a b$. When $\sigma_{2}^{2}=0, \boldsymbol{V}$ will be singular.

$\Delta$

Following Nelder (1965a,b) and Mejza (1992), we say that the mixed model has OBS, when $\boldsymbol{V}$ can be written as

$$
\boldsymbol{V}=\sum_{j=1}^{m^{\circ}} \gamma_{j}^{\circ} \boldsymbol{Q}_{j}^{\circ}
$$

where the $\boldsymbol{Q}_{1}^{\circ}, \ldots, \boldsymbol{Q}_{m}^{\circ}$ are known mutually orthogonal orthogonal projection matrices such that $\sum_{j=1}^{m^{\circ}} \boldsymbol{Q}_{j}^{\circ}=\boldsymbol{I}_{n}$. This means that the matrices in $\mathscr{V}$ have simultaneous spectral decomposition.

The $\gamma_{j}^{\circ}, j=1, \ldots, m^{\circ}$, will be non negative and, when they are positive, $\boldsymbol{V}$ will be positive definite.

We now establish 
Proposition 1 The mixed model

$$
\boldsymbol{y}=\sum_{i=0}^{w} \boldsymbol{X}_{i} \boldsymbol{\beta}_{i},
$$

with $\boldsymbol{\beta}_{0}$ fixed and the $\boldsymbol{\beta}_{1}, \ldots, \boldsymbol{\beta}_{w}$ independent with null mean vectors and variancecovariance matrices $\sigma_{i}^{2} \boldsymbol{I}_{c_{i}}, i=1, \ldots, w$, has $O B S$ when and only when the matrices $\boldsymbol{M}_{1}, \ldots, \boldsymbol{M}_{w}$ commute and

$$
R\left(\left[\boldsymbol{X}_{1} \cdots \boldsymbol{X}_{w}\right]\right)=\mathbb{R}^{n} .
$$

Proof Let us assume that the matrices $\boldsymbol{M}_{1}, \ldots, \boldsymbol{M}_{w}$ commute and that

$$
R\left(\left[\boldsymbol{X}_{1} \cdots \boldsymbol{X}_{w}\right]\right)=\mathbb{R}^{n} .
$$

Then the $\boldsymbol{M}_{1}, \ldots, \boldsymbol{M}_{w}$ will generate a CJAS, $\mathscr{A}(\mathscr{M})$. With $\left\{\boldsymbol{Q}_{1}^{\circ}, \ldots, \boldsymbol{Q}_{m^{\circ}}^{\circ}\right\}=$ $p b(\mathscr{A}(\mathscr{M}))$, we will have $\boldsymbol{M}_{i}=\sum_{j=1}^{m^{\circ}} b_{i, j}^{\circ} \boldsymbol{Q}_{j}^{\circ}, i=1, \ldots, w$, as well as $\boldsymbol{V}=$ $\sum_{i=1}^{w} \sigma_{i}^{2} \boldsymbol{M}_{i}=\sum_{j=1}^{m^{\circ}} \gamma_{j}^{\circ} \boldsymbol{Q}_{j}^{\circ}$, where $\gamma_{j}^{\circ}=\sum_{i=1}^{w} b_{i, j}^{\circ} \sigma_{i}^{2}, j=1, \ldots, m^{\circ}$. Moreover $\sum_{i=1}^{w} \boldsymbol{M}_{i}=\left[\boldsymbol{X}_{1} \cdots \boldsymbol{X}_{w}\right]\left[\boldsymbol{X}_{1} \cdots \boldsymbol{X}_{w}\right]^{\top}$, thus $R\left(\sum_{i=1}^{w} \boldsymbol{M}_{i}\right)=R\left(\left[\boldsymbol{X}_{1} \cdots \boldsymbol{X}_{w}\right]\right)=$ $\mathbb{R}^{n}$, and so $\mathscr{A}(\mathscr{M})$ contains the invertible matrix $\sum_{i=1}^{w} \boldsymbol{M}_{i}$ and so $\sum_{j=1}^{m^{\circ}} \boldsymbol{Q}_{j}^{\circ}=\boldsymbol{I}_{n}$, which establishes the thesis.

Inversely let us assume the model to be OBS so that, whatever $\sigma_{1}^{2}, \ldots, \sigma_{w}^{2}$, we have

$$
\sum_{i=1}^{w} \sigma_{i}^{2} \boldsymbol{M}_{i}=\sum_{j=1}^{m^{\circ}} \gamma_{j}^{\circ} \boldsymbol{Q}_{j}^{\circ},
$$

where the $\boldsymbol{Q}_{1}^{\circ}, \ldots, \boldsymbol{Q}_{m}^{\circ}$ are mutually orthogonal orthogonal projection matrices such that $\sum_{j=1}^{m^{\circ}} \boldsymbol{Q}_{j}^{\circ}=\boldsymbol{I}_{n}$. Then $\mathscr{Q}^{\circ}=\left\{\boldsymbol{Q}_{1}^{\circ}, \ldots, \boldsymbol{Q}_{m}^{\circ}\right\}$ will be the principal basis of a CJAS, $\mathscr{A}^{\circ}$. Then the $\boldsymbol{M}_{1}, \ldots, \boldsymbol{M}_{w}$ will belong to $\mathscr{A}^{\circ}$ and so they commute which completes the proof.

During the proof of Proposition 1 we showed that if the matrices $\boldsymbol{M}_{1}, \ldots, \boldsymbol{M}_{w}$ commute we have

$$
\boldsymbol{M}_{i}=\sum_{j=1}^{m^{\circ}} b_{i, j}^{\circ} \boldsymbol{Q}_{j}^{\circ}, \quad i=1, \ldots, w
$$

where the $\boldsymbol{Q}_{1}^{\circ}, \ldots, \boldsymbol{Q}_{m}^{\circ}$ are mutually orthogonal orthogonal projection matrices, and that

$$
\boldsymbol{V}=\sum_{j=1}^{m^{\circ}} \gamma_{j}^{\circ} \boldsymbol{Q}_{j}^{\circ}
$$

with

$$
\gamma_{j}^{\circ}=\sum_{i=1}^{w} b_{i, j}^{\circ} \sigma_{i}^{2}, \quad j=1, \ldots, m^{\circ} .
$$


When $R\left(\left[\boldsymbol{X}_{1} \cdots \boldsymbol{X}_{w}\right]\right)=\mathbb{R}^{n}$, the $\boldsymbol{Q}_{1}^{\circ}, \ldots, \boldsymbol{Q}_{m}^{\circ}$ 。 constitute the principal basis of a complete CJAS.

We point out that since matrices $\boldsymbol{M}_{i}, i=1, \ldots, w$, are positive semi-definite, we have $b_{i, j}^{\circ} \geq 0, j=1, \ldots, m^{\circ}, i=1, \ldots, w$, and so $\gamma_{i, j}^{\circ} \geq 0, j=1, \ldots, m^{\circ}$. When $\sigma_{i}^{2}>0, i=1, \ldots, w$, we have $\gamma_{j}^{\circ}>0, j=1, \ldots, m^{\circ}$, and $\boldsymbol{V}$ will be positive definite.

We may take

$$
\boldsymbol{B}^{\circ}=\left[b_{i, j}^{\circ}\right]
$$

and so, with

$$
\boldsymbol{\gamma}^{\circ}=\left[\begin{array}{c}
\gamma_{1}^{\circ} \\
\vdots \\
\gamma_{m^{\circ}}^{\circ}
\end{array}\right], \quad \boldsymbol{\sigma}^{2}=\left[\begin{array}{c}
\sigma_{1}^{2} \\
\vdots \\
\sigma_{w}^{2}
\end{array}\right]
$$

we have

$$
\boldsymbol{\gamma}^{\circ}=\boldsymbol{B}^{\circ^{\top}} \boldsymbol{\sigma}^{2}
$$

When the matrices $\boldsymbol{M}_{1}, \ldots, \boldsymbol{M}_{w}$ are linearly independent, the row vectors of $\boldsymbol{B}^{\circ}$, which are the column vectors of $\boldsymbol{B}^{{ }^{\top}}$, are linearly independent, and

$$
\sigma^{2}=L^{\circ} \gamma^{\circ}
$$

where $\boldsymbol{L}^{\circ}$ is a left inverse of $\boldsymbol{B}^{\circ}$. When $m^{\circ}>w=\operatorname{rank}\left(\boldsymbol{B}^{\circ}\right)$ there are more left inverses of $\boldsymbol{B}^{\circ^{\top}}$, one of them being the Moore-Penrose inverse, $\left(\boldsymbol{B}^{\circ^{\top}}\right)^{\dagger}$.

$\nabla$

For instance, for the two factor model we've considered above we have

$$
\boldsymbol{B}^{\circ}=\left[\begin{array}{ll}
a & 0 \\
1 & 1
\end{array}\right],
$$

which is an invertible matrix and

$$
\left\{\begin{array}{l}
\gamma_{1}^{\circ}=a \sigma_{1}^{2}+\sigma_{2}^{2} \\
\gamma_{2}^{\circ}=\sigma_{2}^{2}
\end{array} .\right.
$$

This model has OBS, since the matrices

$$
\left\{\begin{array}{l}
\boldsymbol{M}_{1}=\boldsymbol{J}_{a} \otimes \boldsymbol{I}_{b} \\
\boldsymbol{M}_{2}=\boldsymbol{I}_{a} \otimes \boldsymbol{I}_{b}
\end{array},\right.
$$


commute. Putting

$$
\boldsymbol{K}_{a}=\boldsymbol{I}_{a}-\frac{1}{a} \boldsymbol{J}_{a}
$$

we now have

$$
\left\{\begin{array}{l}
\boldsymbol{M}_{1}=a \boldsymbol{Q}_{1}^{\circ} \\
\boldsymbol{M}_{2}=\boldsymbol{Q}_{1}^{\circ}+\boldsymbol{Q}_{2}^{\circ}
\end{array}\right.
$$

with

$$
\left\{\begin{array}{l}
\boldsymbol{Q}_{1}^{\circ}=\frac{1}{a} \boldsymbol{J}_{a} \otimes \boldsymbol{I}_{b} \\
\boldsymbol{Q}_{2}^{\circ}=\boldsymbol{K}_{a} \otimes \boldsymbol{I}_{b}
\end{array}\right.
$$

A model with OBS has COBS, if the orthogonal projection matrix, $\boldsymbol{T}$, on the space $\Omega=R\left(\boldsymbol{X}_{0}\right)$, spanned by $\boldsymbol{\mu}$, commutes with the $\boldsymbol{Q}_{1}^{\circ}, \ldots, \boldsymbol{Q}_{m^{\circ}}^{\circ}$.

We now may establish the following proposition.

Proposition 2 The model has COBS if and only if the matrices $\boldsymbol{M}_{1}, \ldots, \boldsymbol{M}_{w}$ and $\boldsymbol{T}$ commute and $R\left(\left[\boldsymbol{X}_{1} \cdots \boldsymbol{X}_{w}\right]\right)=\mathbb{R}^{n}$.

Proof If matrices $\boldsymbol{M}_{1}, \ldots, \boldsymbol{M}_{w}$ and $\boldsymbol{T}$ commute and $R\left(\left[\boldsymbol{X}_{1} \cdots \boldsymbol{X}_{w}\right]\right)=\mathbb{R}^{n}$, according to Proposition 1, the model will have OBS and, due to Lemma $1, \boldsymbol{T}$ commutes with $\boldsymbol{Q}_{1}^{\circ}, \ldots, \boldsymbol{Q}_{w^{\circ}}^{\circ}$ and so the models has COBS.

Now, if the model has COBS, it has OBS, so, again according to Proposition 1, matrices $\boldsymbol{M}_{1}, \ldots, \boldsymbol{M}_{w}$ commute and $R\left(\left[\boldsymbol{X}_{1} \cdots \boldsymbol{X}_{w}\right]\right)=\mathbb{R}^{n}$, which completes the proof.

Let $\mathscr{A}$ be the CJAS generated by the $\boldsymbol{M}_{1}, \ldots, \boldsymbol{M}_{w}$ and $\boldsymbol{T}$, assuming that these matrices commute. Since $\boldsymbol{I}_{n} \in \mathscr{A}(\mathscr{M}) \subset \mathscr{A}, \mathscr{A}$ will be complete, containing $\boldsymbol{T}$, $\boldsymbol{T}^{c}=\boldsymbol{I}_{n}-\boldsymbol{T}, \boldsymbol{Q}_{1}^{\circ}, \ldots, \boldsymbol{Q}_{m^{\circ}}^{\circ}$ and the products $\boldsymbol{T} \boldsymbol{Q}_{j}^{\circ}$ and $\boldsymbol{T}^{c} \boldsymbol{Q}_{j}^{\circ}, j=1, \ldots, m^{\circ}$. Those of these product matrices that are non null will be mutually orthogonal orthogonal projection matrices so they will constitute the principal basis of a CJAS, $\mathscr{A}^{\circ \circ}$, containing $\boldsymbol{Q}_{1}^{\circ}, \ldots, \boldsymbol{Q}_{m^{\circ}}^{\circ}, \boldsymbol{M}_{1}, \ldots, \boldsymbol{M}_{w}$ and $\boldsymbol{T}$.

We now establish the equivalence

Proposition $3 \mathscr{A}=\mathscr{A}^{\circ \circ}$.

Proof Since $\boldsymbol{M}_{1}, \ldots, \boldsymbol{M}_{w}, \boldsymbol{T} \in \mathscr{A}^{\circ \circ}$, we have $\mathscr{A} \subseteq \mathscr{A}^{\circ \circ}$. Inversely, the matrices in $p b\left(\mathscr{A}^{\circ \circ}\right)$ belong to $\mathscr{A}$, so $\mathscr{A}^{\circ \circ} \subseteq \mathscr{A}$. 
When, with a given $c$,

$$
\boldsymbol{T} \prod_{i=1}^{w} \boldsymbol{M}_{i}=c \boldsymbol{J}_{n}
$$

we will have $\frac{1}{n} \boldsymbol{J}_{n} \in \mathscr{A}$ and $\mathscr{A}$ is regular.

$\nabla$

In the two factor model that we considered before,

$$
\boldsymbol{T}=\boldsymbol{I}_{a} \otimes \frac{1}{b} \boldsymbol{J}_{b},
$$

so we get the

$$
\left\{\begin{array} { l } 
{ \boldsymbol { Q } _ { 1 } = \boldsymbol { T } \boldsymbol { Q } _ { 1 } ^ { \circ } = \frac { 1 } { a } \boldsymbol { J } _ { a } \otimes \frac { 1 } { b } \boldsymbol { J } _ { b } } \\
{ \boldsymbol { Q } _ { 2 } = \boldsymbol { T } \boldsymbol { Q } _ { 2 } ^ { \circ } = \boldsymbol { K } _ { a } \otimes \frac { 1 } { b } \boldsymbol { J } _ { b } }
\end{array} \quad \left\{\begin{array}{l}
\boldsymbol{Q}_{3}=\boldsymbol{T}^{c} \boldsymbol{Q}_{1}^{\circ}=\frac{1}{a} \boldsymbol{J}_{a} \otimes \boldsymbol{K}_{b} \\
\boldsymbol{Q}_{4}=\boldsymbol{T}^{c} \boldsymbol{Q}_{2}^{\circ}=\boldsymbol{K}_{a} \otimes \boldsymbol{K}_{b}
\end{array}\right.\right.
$$

with $\boldsymbol{K}_{b}=\boldsymbol{I}_{b}-\frac{1}{b} \boldsymbol{J}_{b}$, since

$$
\boldsymbol{T}^{c}=\boldsymbol{I}_{a} \otimes \boldsymbol{K}_{b}
$$

Now, for this model,

$$
\boldsymbol{T} \boldsymbol{M}_{1} \boldsymbol{M}_{2}=a \frac{1}{a} \boldsymbol{J}_{a} \otimes \frac{1}{b} \boldsymbol{J}_{b}=\boldsymbol{J}_{a} \otimes \frac{1}{b} \boldsymbol{J}_{b},
$$

so $\mathscr{A}^{\circ}$ will be regular. Moreover, since $\mathscr{A}^{\circ} \subset \mathscr{A}$, we also will have $\frac{1}{n} J_{n} \in \mathscr{A}$ and $\mathscr{A}$ is also regular.

As in this example we may order the matrices in $p b(\mathscr{A})$ to have $\boldsymbol{Q}_{1}, \ldots, \boldsymbol{Q}_{z}\left[\boldsymbol{Q}_{z+1}\right.$, $\left.\ldots, \boldsymbol{Q}_{m}\right]$ as the non null matrices $\boldsymbol{T} \boldsymbol{Q}_{j}^{\circ}\left[\boldsymbol{T}^{c} \boldsymbol{Q}_{j}^{\circ}\right], j=1, \ldots, m^{\circ}$.

Let $z^{\circ} \geq 0$ be the number of matrices $Q_{j}^{\circ}$ such that

$$
\boldsymbol{T} \boldsymbol{Q}_{j}^{\circ}=\boldsymbol{Q}_{j}^{\circ}, \quad j=1, \ldots, m^{\circ}
$$

We may then put

$$
\begin{cases}\boldsymbol{Q}_{j}=\boldsymbol{T} \boldsymbol{Q}_{j}^{\circ}=\boldsymbol{Q}_{j}^{\circ}, & j=1, \ldots, z^{\circ} \\ \boldsymbol{Q}_{j}=\boldsymbol{T} \boldsymbol{Q}_{j}^{\circ} \neq \boldsymbol{Q}_{j}^{\circ}, & j=z^{\circ}+1, \ldots, z . \\ \boldsymbol{Q}_{j}=\boldsymbol{T}^{c} \boldsymbol{Q}_{j-z+z^{\circ}}^{\circ} & j=z+1 \ldots, m\end{cases}
$$


So,

$$
\begin{cases}\boldsymbol{Q}_{j}^{\circ}=\boldsymbol{Q}_{j}, & j=1, \ldots, z^{\circ} \\ \boldsymbol{Q}_{j}^{\circ}=\boldsymbol{Q}_{j}+\boldsymbol{Q}_{j+z-z^{\circ},} & j=z^{\circ}+1, \ldots, z \\ \boldsymbol{Q}_{j}^{\circ}=\boldsymbol{Q}_{j+z-z^{\circ},} & j=z+1, \ldots, m^{\circ}\end{cases}
$$

and $\boldsymbol{T}=\sum_{j=1}^{z} \boldsymbol{Q}_{j}$.

Since $\boldsymbol{M}_{1}, \ldots, \boldsymbol{M}_{w} \in \mathscr{A}$ we will have

$$
\boldsymbol{M}_{i}=\sum_{j=1}^{m} b_{i, j} \boldsymbol{Q}_{j}, \quad i=1, \ldots, w
$$

and so

$$
\boldsymbol{V}=\sum_{j=1}^{m} \gamma_{j} \boldsymbol{Q}_{j}
$$

with $\gamma_{j}=\sum_{i=1}^{w} b_{i, j} \sigma_{i}^{2}, j=1, \ldots, m$. Putting

$$
\left\{\begin{array}{l}
\boldsymbol{B}=\left[b_{i, j}\right]=[\boldsymbol{B}(1) \vdots \boldsymbol{B}(2)] \\
\boldsymbol{\gamma}(1)=\left[\begin{array}{c}
\gamma_{1} \\
\vdots \\
\gamma_{z}
\end{array}\right] \quad ; \quad \gamma(2)=\left[\begin{array}{c}
\gamma_{z+1} \\
\vdots \\
\gamma_{m}
\end{array}\right],
\end{array}\right.
$$

where $\boldsymbol{B}(1)$ has $z$ columns, we get

$$
\boldsymbol{\gamma}(\ell)=\boldsymbol{B}(\ell)^{\top} \boldsymbol{\sigma}^{2}, \quad \ell=1,2 .
$$

When the matrices $\boldsymbol{M}_{1}, \ldots, \boldsymbol{M}_{w}$ are linearly independent, so are the row vectors of $\boldsymbol{B}$.

Now,

$$
\begin{aligned}
\boldsymbol{V} & =\sum_{j=1}^{z^{\circ}} \gamma_{j}^{\circ} \boldsymbol{Q}_{j}^{\circ}+\sum_{j=z^{\circ}+1}^{z} \gamma_{j}^{\circ} \boldsymbol{Q}_{j}^{\circ}+\sum_{j=z+1}^{m^{\circ}} \gamma_{j}^{\circ} \boldsymbol{Q}_{j}^{\circ} \\
& =\sum_{j=1}^{z^{\circ}} \gamma_{j}^{\circ} \boldsymbol{Q}_{j}+\sum_{j=z^{\circ}+1}^{z} \gamma_{j}^{\circ}\left(\boldsymbol{Q}_{j}+\boldsymbol{Q}_{j+z-z^{\circ}}\right)+\sum_{j=z+1}^{m^{\circ}} \gamma_{j}^{\circ} \boldsymbol{Q}_{j+z-z^{\circ}} \\
& =\sum_{j=1}^{m} \gamma_{j} \boldsymbol{Q}_{j},
\end{aligned}
$$

with 


$$
\begin{cases}\gamma_{j}=\gamma_{j}^{\circ}, & j=1, \ldots, z^{\circ} \\ \gamma_{j}=\gamma_{j+z-z^{\circ}}=\gamma_{j}^{\circ}, & j=z^{\circ}+1, \ldots, z \\ \gamma_{j+z-z^{\circ}}=\gamma_{j}^{\circ}, & j=z+1, \ldots, m^{\circ}\end{cases}
$$

Thus the rows of $\boldsymbol{B}^{\top}$ with indexes $j$ and $j+z-z^{\circ}, j=z^{\circ}+1, \ldots, z$ are identical and

$$
\gamma=\left[\begin{array}{l}
\gamma(1) \\
\gamma(2)
\end{array}\right]=C \gamma^{\circ},
$$

with

$$
\boldsymbol{C}=\left[\begin{array}{ccc}
\boldsymbol{I}_{z^{\circ}} & 0 & 0 \\
0 & \boldsymbol{I}_{z-z^{\circ}} & 0 \\
0 & \boldsymbol{I}_{z-z^{\circ}} & 0 \\
0 & 0 & \boldsymbol{I}_{m-z}
\end{array}\right]
$$

When $z^{\circ}=0\left[m=2 z-z^{\circ}\right]$ the first [last] row and column of sub-matrices must be deleted.

$\nabla$

Namely, in the two factor model, we have $z^{\circ}=0$ and $m=2 z-z^{\circ}$,

$$
\left\{\begin{array}{l}
\gamma_{1}=\gamma_{3}=\gamma_{1}^{\circ}=a \sigma_{1}^{2}+\sigma_{2}^{2} \\
\gamma_{2}=\gamma_{4}=\gamma_{2}^{\circ}=\sigma_{2}^{2}
\end{array},\right.
$$

and $\boldsymbol{C}=\mathbf{1}_{2} \otimes \boldsymbol{I}_{2}$ as well as $\boldsymbol{B}(1)=\boldsymbol{B}(2)=\boldsymbol{B}^{\circ}$.

The $\gamma_{1}, \ldots, \gamma_{z}, \gamma_{z+1}, \ldots, \gamma_{m}$ will be the canonical variance components.

As we shall see, we only have unbiased estimators for the $\gamma_{z+1}, \ldots, \gamma_{m}$ unless we introduce additional assumptions.

When the row vectors of $\boldsymbol{B}(2)$, which are the columns vectors of $\boldsymbol{B}(2)^{\top}$, are linearly independent, we have, with $\boldsymbol{L}$ a left inverse of $\boldsymbol{B}(2)^{\top}$,

$$
\boldsymbol{\sigma}^{2}=\boldsymbol{L} \boldsymbol{\gamma}(2)
$$

as well as

$$
\boldsymbol{\gamma}(1)=\boldsymbol{B}(1)^{\top} \boldsymbol{L} \boldsymbol{\gamma}(2)
$$

and we also have unbiased estimators for the usual variance components, see e.g. Fonseca et al. (2003) and Carvalho et al. (2008). These estimators will depend on 
which left inverse is chosen. We now discuss that choice starting by establishing the following lemma.

Lemma 2 If $\boldsymbol{W}$ is a positive semi-definite matrix, we have $\boldsymbol{W}=\boldsymbol{W}^{\frac{1}{2}} \boldsymbol{W}^{\frac{1}{2}}$, with $\boldsymbol{W}^{\frac{1}{2}} a$ positive semi-definite matrix and

$$
\left\|\boldsymbol{C W} \boldsymbol{C}^{\top}\right\| \leq\left\|\boldsymbol{W}^{\frac{1}{2}}\right\|^{2}\left\|\boldsymbol{C}^{\top} \boldsymbol{C}\right\|
$$

Proof When $\boldsymbol{B}$ is a $k \times k$ positive semi-definitive matrix, where $\|\boldsymbol{A}\|$ represents the euclidean matrix norm, we have $\boldsymbol{W}=\boldsymbol{P}^{\top} \boldsymbol{D}\left(r_{1} \ldots r_{k}\right) \boldsymbol{P}$ where $\boldsymbol{P}$ is an orthogonal matrix and $\boldsymbol{D}\left(r_{1} \ldots r_{k}\right)$ is a diagonal matrix whose principal elements, $r_{1}, \ldots, r_{k}$, are non negative eigenvalues of $\boldsymbol{B}$. Thus $\boldsymbol{W}=\boldsymbol{W}^{\frac{1}{2}} \boldsymbol{W}^{\frac{1}{2}}$ with $\boldsymbol{W}^{\frac{1}{2}}=\boldsymbol{P}^{\top} \boldsymbol{D}\left(r_{1}^{\frac{1}{2}} \ldots r_{k}^{\frac{1}{2}}\right) \boldsymbol{P}$.

Now $\left\|\boldsymbol{M} \boldsymbol{M}^{\top}\right\|=\left\|\boldsymbol{M}^{\top} \boldsymbol{M}\right\|$ so, since the euclidean norm is a matrix norm, see Schott (1997), we have

$$
\left\|\boldsymbol{C} \boldsymbol{W} \boldsymbol{C}^{\top}\right\|=\left\|\boldsymbol{C} \boldsymbol{W}^{\frac{1}{2}} \boldsymbol{W}^{\frac{1}{2}} \boldsymbol{C}^{\top}\right\|=\left\|\boldsymbol{W}^{\frac{1}{2}} \boldsymbol{C}^{\top} \boldsymbol{C} \boldsymbol{W}^{\frac{1}{2}}\right\| \leq\left\|\boldsymbol{W}^{\frac{1}{2}}\right\|\left\|\boldsymbol{C}^{\top} \boldsymbol{C}\right\|\left\|\boldsymbol{W}^{\frac{1}{2}}\right\|
$$

and the thesis is established.

The single value decomposition of $\boldsymbol{B}(2)^{\top}$ gives, see Schott (1997),

$$
\boldsymbol{B}(2)^{\top}=\boldsymbol{P}^{\top}\left[\begin{array}{l}
\boldsymbol{\Delta} \\
\mathbf{0}
\end{array}\right] \boldsymbol{Q},
$$

with $\boldsymbol{P}$ and $\boldsymbol{Q}$ orthogonal matrices, $\boldsymbol{\Delta}$ diagonal invertible matrix and $\mathbf{0}$ the null matrix. If $\boldsymbol{L}$ is a left inverse of $\boldsymbol{B}(2)^{\top}$ we have

$$
\boldsymbol{L}=\boldsymbol{Q}^{\top}\left[\boldsymbol{\Delta}^{-1} \boldsymbol{U}\right] \boldsymbol{P},
$$

where $\boldsymbol{U}$ may be any $m \times(m-z-w)$ matrix, while

$$
\boldsymbol{L}^{\dagger}=\left(\boldsymbol{B}(2)^{\top}\right)^{\dagger}=\boldsymbol{Q}^{\top}\left[\boldsymbol{\Delta}^{-1} \mathbf{0}\right] \boldsymbol{P}
$$

Now $\widetilde{\gamma}(2)$ has the variance-covariance matrix

$$
\boldsymbol{V}=\boldsymbol{D}\left(2 \frac{\gamma_{z+1}^{2}}{g_{z+1}} \ldots 2 \frac{\gamma_{m}^{2}}{g_{m}}\right)
$$

with $g_{j}=\operatorname{rank}\left(\boldsymbol{Q}_{j}\right), j=z+1, \ldots, m$, so

$$
\left\|\boldsymbol{V}^{\frac{1}{2}}\right\|^{2}=2 \sum_{j=z+1}^{m} \frac{\gamma_{j}^{2}}{g_{j}}
$$

and we get the upper bound 


$$
\left\|\boldsymbol{L} \boldsymbol{V} \boldsymbol{L}^{\top}\right\|^{2} \leq\left(2 \sum_{j=z+1}^{m} \frac{\gamma_{j}^{2}}{g_{j}}\right)\left\|\boldsymbol{L}^{\top} \boldsymbol{L}\right\|,
$$

where

$$
\boldsymbol{L}^{\top} \boldsymbol{L}=\boldsymbol{P}^{\top}\left[\begin{array}{cc}
\boldsymbol{\Delta}^{-2} & \boldsymbol{\Delta}^{-1} \boldsymbol{U} \\
\boldsymbol{U}^{\top} \boldsymbol{\Delta}^{-1} & \boldsymbol{U}^{\top} \boldsymbol{U}
\end{array}\right] \boldsymbol{P} .
$$

Since

$$
\left\|\boldsymbol{L}^{\dagger^{\top}} \boldsymbol{L}^{\dagger}\right\| \leq\left\|\boldsymbol{L}^{\top} \boldsymbol{L}\right\|
$$

we established the following theorem.

Theorem 1 The Moore-Penrose inverse minimizes the upper bound for the euclidean norm of the variance-covariance matrix of the unbiased estimator $\widetilde{\boldsymbol{\sigma}}^{2}=\boldsymbol{L} \widetilde{\boldsymbol{\gamma}}(2)$, where $\boldsymbol{L}$ is a left inverse of $\boldsymbol{B}(2)^{\top}$.

Thus we will choose $\boldsymbol{L}=\boldsymbol{L}^{\dagger}$ in order to obtain unbiased estimators for $\boldsymbol{\sigma}^{2}$. In this case $\boldsymbol{\gamma}(2)$ and $\boldsymbol{\sigma}^{2}$ determine each other. These are the relevant parameters for the random effects part of the model given by the orthogonal projection of $\boldsymbol{y}$ on the orthogonal complement $\Omega^{\perp}$ of $\Omega$, since the corresponding mean vector will be null and, with $\boldsymbol{T}^{c}=\boldsymbol{I}_{n}-\boldsymbol{T}$, we have the variance-covariance matrix

$$
\boldsymbol{T}^{c} \boldsymbol{V} \boldsymbol{T}^{c}=\sum_{i=1}^{w} \sigma_{i}^{2} \boldsymbol{T}^{c} \boldsymbol{M}_{i} \boldsymbol{T}^{c}=\sum_{j=z+1}^{m} \gamma_{j} \boldsymbol{Q}_{j}
$$

We thus say that the random effects part segregates itself as a sub-model and that we have a segregated COBS, S-COBS. The existence of segregation allows us to estimate all canonical and usual variance components.

$\nabla$

In the two factor model, we have $w=z=2$ and $m=4$ as well as

$$
\boldsymbol{B}(1)=\boldsymbol{B}(2)=\left[\begin{array}{ll}
a & 0 \\
1 & 1
\end{array}\right]
$$

since

$$
\left\{\begin{array}{l}
\boldsymbol{M}_{1}=a \boldsymbol{Q}_{1}+a \boldsymbol{Q}_{3} \\
\boldsymbol{M}_{2}=\boldsymbol{Q}_{1}+\boldsymbol{Q}_{2}+\boldsymbol{Q}_{3}+\boldsymbol{Q}_{4}
\end{array} .\right.
$$

Thus, in this case we have a S-COBS. 
Another special case of interest is when $z^{\circ}=0$, and so

$$
\begin{cases}\boldsymbol{Q}_{j}=\boldsymbol{T} \boldsymbol{Q}_{j}^{\circ}, & j=1, \ldots, z \\ \boldsymbol{Q}_{j+z}=\boldsymbol{T}^{c} \boldsymbol{Q}_{j}^{\circ}, & j=1, \ldots, z\end{cases}
$$

so

$$
\left\{\begin{array}{ll}
\boldsymbol{Q}_{j}^{\circ}=\boldsymbol{Q}_{j}+\boldsymbol{Q}_{j+z}, & j=1, \ldots, z \\
\gamma_{j}^{\circ}=\gamma_{j}=\gamma_{j+z}, & j=1, \ldots, z
\end{array},\right.
$$

then $\boldsymbol{\gamma}(1)$ will be a sub-vector of $\boldsymbol{\gamma}(2)$ and its components are estimated simultaneously with the components of $\boldsymbol{\gamma}(2)$.

In this case we have the pairs $\left(\boldsymbol{Q}_{j}, \boldsymbol{Q}_{j+z}\right)$ of matrices and the pairs $\left(\gamma_{j}, \gamma_{j+z}\right)$ of canonical variance components, $j=1, \ldots, z$. This pairing is of course partial unless $m=2 z$, but we will call these COBS as paired, P-COBS. As stated above the condition for having a P-COBS is that $z^{\circ}=0$ and we can define them more precisely saying that P-COBS are COBS for which $z^{\circ}=0$. This means that there is no $R\left(\boldsymbol{Q}_{j}^{\circ}\right)$ strictly contained in $\Omega=R\left(\boldsymbol{X}_{0}\right)$.

\section{$\nabla$}

In the two factor model we have been considering, we have $z=2, z^{\circ}=0$ as well as

$$
\left\{\begin{array}{l}
Q_{1}^{\circ}=Q_{1}+Q_{2} \\
Q_{2}^{\circ}=Q_{2}+Q_{4}
\end{array}\right.
$$

and, as we saw,

$$
\left\{\begin{array}{l}
\gamma_{1}^{\circ}=\gamma_{1}=\gamma_{3} \\
\gamma_{2}^{\circ}=\gamma_{2}=\gamma_{4}
\end{array}\right.
$$

so that model is simultaneously S-COBS and P-COBS.

When there is pairing, $\boldsymbol{B}(1)$ is a sub-matrix of $\boldsymbol{B}(2)$, so the row vector of $\boldsymbol{B}$ are linearly independent when and only when the row vectors of $\boldsymbol{B}(2)$ are linearly independent. Thus, if matrices $\boldsymbol{M}_{1}, \ldots, \boldsymbol{M}_{w}$ are linearly independent, the column vectors of $\boldsymbol{B}(2)^{\top}$ will be linearly independent, and

$$
\boldsymbol{\sigma}^{2}=\left(\boldsymbol{B}(2)^{\top}\right)^{\dagger} \boldsymbol{\gamma}(2)
$$

while $\boldsymbol{\gamma}(1)$ will be a sub-vector of $\boldsymbol{\gamma}(2)$ enabling us to estimate the canonical variance components. 
Thus the linear independence of matrices $\boldsymbol{M}_{1}, \ldots, \boldsymbol{M}_{w}$ and pairing implies segregation.

\section{Inference}

In this section we will consider inference for COBS. Moreover, assuming the normality, we will show how we test hypotheses using pivot variables.

\subsection{Estimation}

Lets assume the mixed model

$$
\boldsymbol{y}=\sum_{i=0}^{w} \boldsymbol{X}_{i} \boldsymbol{\beta}_{i}
$$

to have COBS and use the same notations as before.

With $\boldsymbol{A}_{j}$ a matrix whose row vectors constitute an orthonormal basis for $R\left(\boldsymbol{Q}_{j}\right)$, $j=1, \ldots, m$, we can take

$$
\begin{cases}\boldsymbol{\eta}_{j}=\boldsymbol{A}_{j} \boldsymbol{\mu} & j=1, \ldots, m \\ \widetilde{\boldsymbol{\eta}}_{j}=\boldsymbol{A}_{j} \boldsymbol{y} & j=1, \ldots, m\end{cases}
$$

as well as the sums of squares $S_{j}=\left\|\widetilde{\boldsymbol{\eta}}_{j}\right\|^{2}, j=1, \ldots, m$. So $\boldsymbol{Q}_{j}=\boldsymbol{A}_{j}^{\top} \boldsymbol{A}_{j}, j=$ $1, \ldots, m$, and

$$
\left\{\begin{array}{c}
\boldsymbol{I}_{n}=\sum_{j=1}^{m} \boldsymbol{Q}_{j}=\sum_{j=1}^{m} \boldsymbol{A}_{j}^{\top} \boldsymbol{A}_{j} \\
\boldsymbol{T}=\sum_{j=1}^{z} \boldsymbol{Q}_{j}=\sum_{j=1}^{z} \boldsymbol{A}_{j}^{\top} \boldsymbol{A}_{j}
\end{array}\right.
$$

thus

$$
\left\{\begin{array}{l}
\boldsymbol{y}=\sum_{j=1}^{m} \boldsymbol{A}_{j}^{\top} \boldsymbol{A}_{j} \boldsymbol{y}=\sum_{j=1}^{m} \boldsymbol{A}_{j}^{\top} \widetilde{\boldsymbol{\eta}}_{j} \\
\boldsymbol{\mu}=\sum_{j=1}^{z} \boldsymbol{A}_{j}^{\top} \boldsymbol{A}_{j} \boldsymbol{\mu}=\sum_{j=1}^{z} \boldsymbol{A}_{j}^{\top} \boldsymbol{\eta}_{j}
\end{array},\right.
$$

since $\boldsymbol{\eta}_{j}=0, j=z+1, \ldots, m$.

The expression

$$
\boldsymbol{y}=\sum_{j=1}^{m} \boldsymbol{A}_{j}^{\top} \widetilde{\boldsymbol{\eta}}_{j}
$$

corresponds to the canonical form of the model which will have mean vector and variance-covariance matrix 


$$
\left\{\begin{array}{l}
\boldsymbol{\mu}=\sum_{j=1}^{z} \boldsymbol{A}_{j}^{\top} \boldsymbol{\eta}_{j} \\
\boldsymbol{V}=\sum_{j=1}^{m} \gamma_{j} \boldsymbol{Q}_{j}
\end{array}\right.
$$

The $\widetilde{\boldsymbol{\eta}}_{j}, j=1, \ldots, m$, will have mean vectors $\boldsymbol{\eta}_{j}, j=1, \ldots, m$, variancecovariance matrices

$$
\boldsymbol{A}_{j} \boldsymbol{V} \boldsymbol{A}_{j}^{\top}=\gamma_{j} \boldsymbol{I}_{g_{j}}, \quad j=1, \ldots, m,
$$

where $g_{j}=\operatorname{rank}\left(\boldsymbol{A}_{j}\right), j=1, \ldots, m$, and cross-covariance matrices

$$
\boldsymbol{A}_{j} \boldsymbol{V} \boldsymbol{A}_{j^{\prime}}^{\top}=\mathbf{0}_{g_{j} \times g_{j^{\prime}}}, \quad j \neq j^{\prime},
$$

where $\mathbf{0}_{r \times s}$ is the $r \times s$ null matrix.

Since the matrices $\boldsymbol{T}$ and $\boldsymbol{V}$ commute, the LSE of estimable vectors will be BLUE, see Zmyślony (1980). We know that $\boldsymbol{\Psi}=\boldsymbol{G} \boldsymbol{\mu}$ is estimable if and only if $\boldsymbol{G}=\boldsymbol{U} \boldsymbol{X}_{0}$, see Mexia (1990). Therefore,

$$
\boldsymbol{\Psi}=\boldsymbol{U} \boldsymbol{X}_{0} \boldsymbol{\beta}=\boldsymbol{U} \boldsymbol{\mu}=\boldsymbol{U} \sum_{j=1}^{z} \boldsymbol{A}_{j}^{\top} \boldsymbol{\eta}_{j}=\sum_{j=1}^{z} \boldsymbol{U}_{j} \boldsymbol{\eta}_{j}
$$

where $\boldsymbol{U}_{j}=\boldsymbol{U} \boldsymbol{A}_{j}^{\top}, j=1, \ldots, z$. Thus, the estimable vectors are generalized linear combinations of the $\boldsymbol{\eta}_{j}, j=1, \ldots, z$, which are the canonical estimable vectors and we get the estimators

$$
\widetilde{\boldsymbol{\Psi}}=\sum_{j=1}^{z} \boldsymbol{U}_{j} \widetilde{\boldsymbol{\eta}}_{j}
$$

When the CJAS $\mathscr{A}$ is regular, the row vectors of $\boldsymbol{A}_{j}, j=2, \ldots, m$, will be contrast vectors having null sums of components. Then the components of the canonical estimable vectors $\boldsymbol{\eta}_{j}, j=2, \ldots, m$, will be contrasts on the components of $\boldsymbol{\mu}$.

Moreover, the LSE of $\boldsymbol{\Psi}$ will be $\boldsymbol{\Psi}^{*}=\boldsymbol{G} \boldsymbol{\beta}^{*}$, see Mexia (1990), with

$$
\boldsymbol{\beta}^{*}=\left(\boldsymbol{X}_{0}^{\top} \boldsymbol{X}_{0}\right)^{\dagger} \boldsymbol{X}_{0} \boldsymbol{y}
$$

Since, see again Mexia (1990),

$$
\boldsymbol{T}=\boldsymbol{X}_{0}\left(\boldsymbol{X}_{0}^{\top} \boldsymbol{X}_{0}\right)^{\dagger} \boldsymbol{X}_{0}^{\top}
$$

we get

$$
\boldsymbol{\Psi}^{*}=\boldsymbol{U} \boldsymbol{X}_{0} \boldsymbol{\beta}^{*}=\boldsymbol{U} \boldsymbol{T} \boldsymbol{y}=\boldsymbol{U}\left(\sum_{j=1}^{z} \boldsymbol{A}_{j}^{\top} \boldsymbol{A}_{j}\right) \boldsymbol{y}=\sum_{j=1}^{z} \boldsymbol{U}_{j} \tilde{\boldsymbol{\eta}}_{j}=\tilde{\boldsymbol{\Psi}}
$$

so $\widetilde{\boldsymbol{\Psi}}$ will be BLUE. 
Moreover, since $\boldsymbol{\eta}_{j}=\mathbf{0}, j=z+1, \ldots, m$, we have the unbiased estimators

$$
\widetilde{\gamma}_{j}=\frac{S_{j}}{g_{j}}, \quad j=z+1, \ldots, m,
$$

with $g_{j}=\operatorname{rank}\left(\boldsymbol{Q}_{j}\right), j=z+1, \ldots, m$, which will be the components of the unbiased estimator $\widetilde{\gamma}(2)$ of $\boldsymbol{\gamma}(2)$. If the model is a S-COBS we also have the unbiased estimators

$$
\left\{\begin{array}{l}
\tilde{\boldsymbol{\sigma}}^{2}=\left(\boldsymbol{B}(2)^{\top}\right)^{\dagger} \tilde{\boldsymbol{\gamma}}(2) \\
\tilde{\boldsymbol{\gamma}}(1)=\boldsymbol{B}(1)^{\top}\left(\boldsymbol{B}(2)^{\top}\right)^{\dagger} \tilde{\boldsymbol{\gamma}}(2)
\end{array}\right.
$$

of $\boldsymbol{\sigma}^{2}$ and $\boldsymbol{\gamma}(1)$. Moreover, if the model is P-COBS, $\boldsymbol{\gamma}(1)$ is a sub-vector of $\boldsymbol{\gamma}(2)$, so the corresponding sub-vectors $\widetilde{\gamma}(1)$ of $\widetilde{\gamma}(2)$ will be an unbiased estimator of $\gamma(1)$, and

$$
\widetilde{\gamma}=\left[\begin{array}{ll}
\widetilde{\gamma}(1)^{\top} & \widetilde{\gamma}(2)^{\top}
\end{array}\right]^{\top}
$$

will be an unbiased estimator of $\boldsymbol{\gamma}$, and, if the row vectors of $\boldsymbol{B}$ are linearly independent,

$$
\tilde{\boldsymbol{\sigma}}^{2}=\left(\boldsymbol{B}^{\top}\right)^{\dagger} \tilde{\gamma}
$$

will be an unbiased estimator of $\boldsymbol{\sigma}^{2}$.

$\nabla$

In the two factors model we have been considering, we have $z=2$ and $m=4$ and, with $\boldsymbol{L}_{r}$, the matrix obtained deleting the first row equal to $\frac{1}{\sqrt{r}} \mathbf{1}_{r}^{\top}$ of a $r \times r$ orthogonal matrix, we have

$$
\left\{\begin{array}{l}
\boldsymbol{A}_{1}=\frac{1}{\sqrt{a}} \mathbf{1}_{a}^{\top} \otimes \frac{1}{\sqrt{b}} \mathbf{1}_{b}^{\top} \\
\boldsymbol{A}_{2}=\boldsymbol{L}_{a} \otimes \frac{1}{\sqrt{b}} \mathbf{1}_{b}^{\top}
\end{array}, \quad\left\{\begin{array}{l}
\boldsymbol{A}_{3}=\frac{1}{\sqrt{a}} \mathbf{1}_{a}^{\top} \otimes \boldsymbol{L}_{b} \\
\boldsymbol{A}_{4}=\boldsymbol{L}_{a} \otimes \boldsymbol{L}_{b}
\end{array}\right.\right.
$$

Thus, if

$$
\boldsymbol{G}=\boldsymbol{U} \boldsymbol{X}_{0}=\boldsymbol{U}\left(\boldsymbol{I}_{a} \otimes \mathbf{1}_{b}\right),
$$

we have an estimable vector,

$$
\boldsymbol{\Psi}=\boldsymbol{U}_{1} \boldsymbol{\eta}_{1}+\boldsymbol{U}_{2} \boldsymbol{\eta}_{2},
$$


where $\boldsymbol{U}_{j}=\boldsymbol{U} \boldsymbol{A}_{j}^{\top}, j=1,2$, and

$$
\boldsymbol{\eta}_{1}=\boldsymbol{A}_{1} \boldsymbol{\mu}=\left(\frac{1}{\sqrt{a}} \mathbf{1}_{a}^{\top} \otimes \frac{1}{\sqrt{b}} \mathbf{1}_{b}^{\top}\right)\left(\boldsymbol{\beta}_{0} \otimes \mathbf{1}_{b}\right)=\sqrt{a b} \beta_{0, \bullet}
$$

with $\beta_{0, \bullet}$ the mean of the components of $\boldsymbol{\beta}_{0}$ and

$$
\boldsymbol{\eta}_{2}=\boldsymbol{A}_{2} \boldsymbol{\mu}=\left(\boldsymbol{L}_{a} \otimes \frac{1}{\sqrt{b}} \mathbf{1}_{b}^{\top}\right)\left(\boldsymbol{\beta}_{0} \otimes \mathbf{1}_{b}\right)=\sqrt{b} \boldsymbol{L}_{a} \boldsymbol{\beta}_{0} .
$$

Now the sums of the elements in any row of $\boldsymbol{L}_{a}$ is null, so the components $\eta_{2,1}, \ldots, \eta_{2, a-1}$ of $\boldsymbol{\eta}_{2}$, will be contrasts on the components of $\boldsymbol{\beta}_{0}$.

It may be interesting to point out that

$$
\boldsymbol{\beta}_{0}=\mathbf{1}_{a} \beta_{0, \bullet}+\boldsymbol{L}_{a}^{\top} \boldsymbol{L}_{a} \boldsymbol{\beta}_{0}
$$

where $\mathbf{1}_{a} \beta_{0, \bullet}$ is the orthogonal projection of $\boldsymbol{\beta}_{0}$ on $R\left(\mathbf{1}_{a}\right)$ and $\boldsymbol{L}_{a}^{\top} \boldsymbol{L}_{a} \boldsymbol{\beta}_{0}$ is the orthogonal projection of $\boldsymbol{\beta}_{0}$ on the orthogonal complement $R\left(\mathbf{1}_{a}\right)^{\perp}$ of $R\left(\mathbf{1}_{a}\right)$.

For $\eta_{1}$ and $\eta_{2}$ we have the estimators

$$
\left\{\begin{array}{l}
\widetilde{\boldsymbol{\eta}}_{1}=\sqrt{a b} \widetilde{\beta}_{0}, \bullet \\
\widetilde{\boldsymbol{\eta}}_{2}=\sqrt{b} \boldsymbol{L}_{a} \widetilde{\boldsymbol{\beta}}_{0}
\end{array}\right.
$$

with

$$
\widetilde{\beta}_{0, \bullet}=\frac{1}{a b} \sum_{\ell=1}^{a b} y_{\ell}
$$

and $\widetilde{\boldsymbol{\beta}}_{0}$ the vector with components

$$
\widetilde{\beta}_{0, i}=\frac{1}{b} \sum_{\ell=(i-1) b+1}^{i b} y_{\ell}, \quad i=1, \ldots, a
$$

We also have

$$
\begin{cases}S_{3}=\left\|\boldsymbol{A}_{3} \boldsymbol{y}\right\|^{2}, & g_{3}=b-1 \\ S_{4}=\left\|\boldsymbol{A}_{4} \boldsymbol{y}\right\|^{2}, & g_{4}=(a-1)(b-1)\end{cases}
$$

thus getting, since this model is P-COBS, with $z=2$ and $m=4$,

$$
\left\{\begin{array}{l}
\widetilde{\gamma}_{1}=\widetilde{\gamma}_{3}=\frac{S_{3}}{g_{3}} \\
\widetilde{\gamma}_{2}=\widetilde{\gamma}_{4}=\frac{S_{4}}{g_{4}}
\end{array}\right.
$$


Moreover, using the fact that the model is also S-COBS and $\boldsymbol{B}(2)^{\top}$ is invertible, we also get

$$
\left\{\begin{array}{l}
\tilde{\sigma}_{1}^{2}=\frac{\tilde{\gamma}_{3}-\widetilde{\gamma}_{4}}{a} \\
\tilde{\sigma}_{2}^{2}=\widetilde{\gamma}_{4}
\end{array} .\right.
$$

\subsection{Normality}

When normality is assumed we can use pivot variables to obtain confidence regions and, through duality, test hypotheses. We now point out that, if $\widetilde{W} / \theta$ has a known distribution the quantiles of that distribution may be used to obtain, two-sided or onesided confidence intervals for $\theta$. These confidence intervals can then be used to test hypotheses on $\theta$. As pivot variables of this type we have

- the $\frac{S_{j}}{\gamma_{j}}$ which have chi-square distribution with $g_{j}$ degrees of freedom, $\chi_{g_{j}}^{2}, j=$ $z+1, \ldots, m$

- the $\mathscr{F}_{j, j^{\prime}} / \frac{\gamma_{j}}{\gamma_{j^{\prime}}} ; j, j^{\prime}=z+1, \ldots, m$, where

$$
\mathscr{F}_{j, j^{\prime}}=\frac{g_{j^{\prime}}}{g_{j}} \frac{S_{j}}{S_{j^{\prime}}}, \quad j, j^{\prime}=z+1, \ldots, m,
$$

has central $F$ distributions with $g_{j}$ and $g_{j^{\prime}}$ degrees of freedom, $F\left(\cdot \mid g_{j}, g_{j^{\prime}}\right), j, j^{\prime}=$ $z+1, \ldots, m$. Moreover, when $g_{j^{\prime}}>2$ the mean value of $\mathscr{F}_{j, j^{\prime}}$ is $\frac{g_{j^{\prime}}}{g_{j^{\prime}}-2} \frac{\gamma_{j}}{\gamma_{j^{\prime}}}$, so we have the unbiased estimator

$$
\left(\widetilde{\gamma_{j}}\right)=\frac{g_{j^{\prime}}-2}{g_{j^{\prime}}} \mathscr{F}_{j, j^{\prime}}
$$

These pivot variables are related to the random effects part of the model.

$$
\nabla
$$

For the two factor model that we have been considering, the pivot variables would be,

$-\frac{S_{3}}{\gamma_{3}}$ and $\frac{S_{4}}{\gamma_{4}}$

$-\mathscr{F}_{3,4} / \frac{\gamma_{3}}{\gamma_{4}}$ with

$$
\mathscr{F}_{3,4}=\frac{(a-1)(b-1)}{b-1} \frac{S_{3}}{S_{4}} .
$$

Since $\gamma_{4}=\sigma_{2}^{2}$, using $\frac{S_{4}}{\gamma_{4}}$ we can carry out inference for $\sigma_{2}^{2}$. 
Moreover

$$
\frac{\gamma_{3}}{\gamma_{4}}=a \frac{\sigma_{1}^{2}}{\sigma_{2}^{2}}+1
$$

thus $\frac{\gamma_{3}}{\gamma_{4}}=1$ when and only when $\sigma_{1}^{2}=0$. So to test

$$
H_{0}: \sigma_{1}^{2}=0
$$

we have only to test

$$
H_{0}^{\circ}: \frac{\gamma_{3}}{\gamma_{4}}=1
$$

When $z^{\circ}<j \leq z$, we have $\gamma_{j}=\gamma_{j+z-z^{\circ}}$, thus taking

$$
\left\{\begin{array}{l}
\widetilde{\boldsymbol{\Psi}}=\boldsymbol{W} \widetilde{\boldsymbol{\eta}}_{j} \\
\boldsymbol{\Psi}=\boldsymbol{W} \boldsymbol{\eta}_{j}
\end{array}\right.
$$

we have the pivot variable

$$
\mathscr{F}=\frac{g_{j+z-z^{\circ}}}{r} \frac{(\widetilde{\boldsymbol{\Psi}}-\boldsymbol{\Psi})^{\top}\left(\boldsymbol{W} \boldsymbol{W}^{\top}\right)^{\dagger}(\widetilde{\boldsymbol{\Psi}}-\boldsymbol{\Psi})}{S_{j+z-z^{\circ}}}
$$

with central $F$ distribution with $r=\operatorname{rank}(\boldsymbol{W})$ and $g_{j+z-z^{\circ}}$ degrees of freedom,

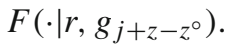

So, with $f_{r, g_{j+z-z^{\circ}, 1-p}}$ the $(1-p)-t h$ quantile of that distribution, we have the $1-p$ level confidence ellipsoid

$$
(\boldsymbol{\Psi}-\widetilde{\boldsymbol{\Psi}})^{\top}\left(\boldsymbol{W} \boldsymbol{W}^{\top}\right)(\boldsymbol{\Psi}-\widetilde{\boldsymbol{\Psi}}) \leq r f_{r, g_{j+z-z^{\circ}, 1-p}} \frac{S_{j+z-z^{\circ}}}{g_{j+z-z^{\circ}}}
$$

for $\boldsymbol{\Psi}$, see Scheffé (1959). We may now test, through duality, hypothesis such as

$$
H_{0}\left(\boldsymbol{\Psi}_{0}\right): \boldsymbol{\Psi}=\boldsymbol{\Psi}_{0}
$$

Of course we may take $\boldsymbol{W}=\boldsymbol{I}_{g_{j}}$ but there are other cases of interest.

$\nabla$

For the two factor model the hypothesis

$$
H_{0}(\mathbf{0}): \boldsymbol{\eta}_{2}=\mathbf{0}
$$


of absence of effects for the fixed factor is highly relevant.

When the model is P-COBS, we can consider this type of pivot variables for all pairs $(j, j+z), j=1, \ldots, z$.

We now point out that

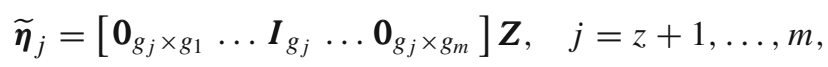

where

$$
\boldsymbol{Z}=\left[\widetilde{\boldsymbol{\eta}}_{z+1}^{\top} \ldots \widetilde{\boldsymbol{\eta}}_{m}^{\top}\right]^{\top}
$$

and that, when normality is assumed, $\boldsymbol{Z}$ is itself normal with density

$$
n(\boldsymbol{z})=\frac{e^{-\frac{1}{2} \sum_{j=z+1}^{m} \frac{s_{j}}{\gamma_{j}}}}{(2 \pi)^{\frac{n^{\circ}}{2}} \prod_{j=z+1}^{m} \gamma_{j}^{\frac{g_{j}}{2}}},
$$

where $n^{\circ}=\sum_{j=z+1}^{m} g_{j}$.

It is easy to see that $S_{z+1}, \ldots, S_{m}$ constitute a sufficient statistic for the family of densities of $\boldsymbol{Z}$. Moreover there are no linear constraints either on the $S_{z+1}, \ldots, S_{m}$ or on the $\gamma_{z+1}^{-1}, \ldots, \gamma_{m}^{-1}$, thus for that family of densities to be full rank exponential its parameter space, see Lehmann and Casella (2003), has to contain a $m-z$ dimensional rectangle, which, since

$$
\boldsymbol{\gamma}(2)=\boldsymbol{B}(2)^{\top} \boldsymbol{\sigma}^{2}
$$

and $R\left(\boldsymbol{B}(2)^{\top}\right)$ has dimension $w$, occurs if and only if

$$
w=m-z,
$$

this is when $\boldsymbol{B}(2)^{\top}$ is invertible.

Then $S_{z+1}, \ldots, S_{m}$ will constitute a complete and sufficient statistic and, accordingly with the Blackwell-Lehman-Scheffé, the estimators we determined for variance-covariance, either usual or canonical, are UMVUE in the class of estimators obtained from $\boldsymbol{Z}$, we will say that they are $\operatorname{UMVUE}(\boldsymbol{Z})$. Namely we will have

$$
\left\{\begin{array}{l}
\tilde{\boldsymbol{\sigma}}^{2}=\left(\boldsymbol{B}(2)^{\top}\right)^{-1} \widetilde{\gamma}(2) \\
\widetilde{\gamma}(1)=\boldsymbol{B}(1)^{\top}\left(\boldsymbol{B}(2)^{\top}\right)^{-1} \widetilde{\gamma}(2)
\end{array}\right.
$$

when $w=m-z$ the matrices

$$
\boldsymbol{W}_{i}=\boldsymbol{M}_{i} \boldsymbol{T}^{c}, \quad i=1, \ldots, w
$$


constitute a basis for the CJAS $\mathscr{A}^{c}$ with principal basis

$$
\boldsymbol{Q}^{c}=\left\{\boldsymbol{Q}_{z+1}, \ldots, \boldsymbol{Q}_{m}\right\}
$$

since

$$
\boldsymbol{W}_{i}=\sum_{j=z+1}^{m} b_{i, j} \boldsymbol{Q}_{j}, \quad i=1, \ldots, w
$$

Inversely, if the $\boldsymbol{W}_{i}, i=1, \ldots, w$, constitute a basis for $\mathscr{A}^{c}$ we will have

$$
w=\operatorname{dim}\left(\mathscr{A}^{c}\right)=m-z
$$

We can now establish the following proposition.

Proposition 4 The $S_{z+1}, \ldots, S_{m}$ constitute a complete and sufficient statistic for the family of densities of $\boldsymbol{Z}$ if and only if $\mathscr{W}=\left\{\boldsymbol{W}_{1}, \ldots, \boldsymbol{W}_{w}\right\}$ is a basis for $\mathscr{A}^{c}$. Then $\widetilde{\gamma}(2), \widetilde{\boldsymbol{\sigma}}^{2}$ and $\widetilde{\boldsymbol{\gamma}}(1)$ will be $\operatorname{UMVUE}(\mathbf{Z})$.

Previously when considering S-COBS we considered for $\boldsymbol{\sigma}^{2}$ estimators

$$
\tilde{\boldsymbol{\sigma}}^{2}=\boldsymbol{L} \tilde{\boldsymbol{\gamma}}(2)
$$

where $\boldsymbol{L}$ is a left inverse of $\boldsymbol{B}(2)^{\top}$. Now $\boldsymbol{B}(2)^{\top}$ may have more that one left inverse so that we may have more than one of these estimators for $\widetilde{\boldsymbol{\sigma}}^{2}$. When normality is assumed, given the estimators

$$
\tilde{\boldsymbol{\sigma}}_{\ell}^{2}=\boldsymbol{L}_{\ell} \widetilde{\boldsymbol{\gamma}}(2), \quad \ell=1,2,
$$

we will have, whatever $\boldsymbol{\gamma}(2)$,

$$
\operatorname{pr}\left(\tilde{\boldsymbol{\sigma}}_{1}^{2}=\tilde{\boldsymbol{\sigma}}_{2}^{2}\right)=1
$$

as well as, with $\boldsymbol{V}\left(\tilde{\boldsymbol{\sigma}}_{\ell}^{2}\right)$, the variance-covariance matrix of $\tilde{\boldsymbol{\sigma}}_{\ell}^{2}, \ell=1,2$,

$$
\boldsymbol{V}\left(\tilde{\boldsymbol{\sigma}}_{1}^{2}\right)=\boldsymbol{V}\left(\tilde{\boldsymbol{\sigma}}_{2}^{2}\right)
$$

Now

$$
\boldsymbol{V}\left(\widetilde{\boldsymbol{\sigma}}_{\ell}^{2}\right)=\boldsymbol{L}_{\ell} \boldsymbol{V}(\widetilde{\boldsymbol{\gamma}}(2)) \boldsymbol{L}_{\ell}^{\top}, \quad \ell=1,2
$$

and $\boldsymbol{V}(\widetilde{\gamma}(2))$, the variance-covariance matrix of $\widetilde{\gamma}(2)$, is diagonal with principal elements $2 \frac{\gamma_{j}^{2}}{g_{j}}, j=z+1, \ldots, m$. Thus these variance-covariance matrices do not depend 
on normality and so $\boldsymbol{V}\left(\tilde{\boldsymbol{\sigma}}_{1}^{2}\right)=\boldsymbol{V}\left(\tilde{\boldsymbol{\sigma}}_{2}^{2}\right)$ even when there is no normality. This reasoning extends to the case of P-COBS and to the estimators

$$
\widetilde{\boldsymbol{\sigma}}^{2}=\boldsymbol{L}^{\circ} \widetilde{\boldsymbol{\gamma}}
$$

where now $\boldsymbol{L}^{\circ}$ is a left inverse of $\boldsymbol{B}^{\top}$. Thus in both cases we may choose, for instance, the Moore-Penrose inverses.

\section{Unbalanced models}

We will now show, with common examples, the use of the previously presented results and so the advantage of their use, specially when computational application may be considered.

\subsection{Only partially balanced nesting}

We start with a simple two factors model with nesting. The first factor will have $a$ levels and fixed effects. The $i$-th level of the first factor will nest $r_{j}$ levels of the second factor, $j=1, \ldots, a$. There will be

$$
n=\sum_{j=1}^{a} r_{j}
$$

observations and the model may be written as

$$
\boldsymbol{y}=\boldsymbol{X}_{0} \boldsymbol{\beta}_{0}+\boldsymbol{X}_{1} \boldsymbol{\beta}_{1}
$$

where

$$
\left\{\begin{array}{l}
\boldsymbol{X}_{0}=\boldsymbol{D}\left(\mathbf{1}_{r_{1}} \ldots \mathbf{1}_{r_{a}}\right) \\
\boldsymbol{X}_{1}=\boldsymbol{I}_{n}
\end{array} .\right.
$$

As before $\boldsymbol{\beta}_{0}$ will be fixed and $\boldsymbol{\beta}_{1}$ will have null mean vector and variancecovariance matrix $\sigma^{2} \boldsymbol{I}_{n}$. Then $w=1, \boldsymbol{Q}_{1}^{\circ}=\boldsymbol{I}_{n}$ and

$$
\left\{\begin{aligned}
\boldsymbol{T} & =\boldsymbol{Q}_{1}=\boldsymbol{D}\left(\frac{1}{r_{1}} \boldsymbol{J}_{r_{1}} \ldots \frac{1}{r_{a}} \boldsymbol{J}_{r_{a}}\right) \\
\boldsymbol{Q}_{2} & =\boldsymbol{D}\left(\boldsymbol{K}_{r_{1}} \ldots \boldsymbol{K}_{r_{a}}\right)
\end{aligned}\right.
$$

with $\boldsymbol{K}_{r_{i}}=\boldsymbol{I}_{r_{i}}-\frac{1}{r_{i}} \boldsymbol{J}_{r_{i}}, i=1, \ldots, a$, clearly $\boldsymbol{M}_{1}=\boldsymbol{I}_{n}$ and $\boldsymbol{T}$ commute, so the model has COBS. Moreover $z^{\circ}=0$, so the model is P-COBS. Since the $r_{1}, \ldots, r_{a}$ may be whatever, the model is unbalanced. 
Let us now consider a design with $w$ levels unbalanced on the last. Given the positive integers $a_{1}, \ldots, a_{w-1}$, let us put

$$
\left\{\begin{array}{l}
b_{0}=1 \\
b_{i}=\prod_{h=1}^{i} a_{h}, i=1, \ldots, w-1
\end{array},\right.
$$

and, with

$$
r_{h}=c_{h} b_{w-1}, \quad h=1, \ldots, n_{0}
$$

take

$$
\left\{\begin{array}{l}
\boldsymbol{X}_{0}=\boldsymbol{D}\left(\mathbf{1}_{r_{1}} \ldots \mathbf{1}_{r_{n_{0}}}\right) \\
\boldsymbol{X}_{i}=\boldsymbol{I}_{n_{i}} \otimes \mathbf{1}_{b_{w-i}}, i=1, \ldots, w
\end{array}\right.
$$

where

$$
\left\{\begin{array}{l}
n_{1}=\sum_{h=1}^{n_{0}} c_{h} \\
n_{i}=a_{w+1-i} n_{i-1}, i=2, \ldots, w
\end{array}\right.
$$

The total number of observations will be $n=\sum_{h=1}^{n_{0}} r_{h}$.

So we will have the orthogonal projection matrices on the

$$
\Omega_{\ell}=R\left(\boldsymbol{X}_{\ell}\right), \quad \ell=0, \ldots, w,
$$

given by

$$
\left\{\begin{array}{l}
\boldsymbol{P}_{0}=\boldsymbol{D}\left(\frac{1}{r_{1}} \boldsymbol{J}_{r_{1}} \ldots \frac{1}{r_{n_{0}}} \boldsymbol{J}_{r_{n_{0}}}\right) \\
\boldsymbol{P}_{i}=\boldsymbol{I}_{n_{i}} \otimes \frac{1}{b_{w-i}} \boldsymbol{J}_{b_{w-i}}, i=1, \ldots, w-1 \\
\boldsymbol{P}_{w}=\boldsymbol{I}_{n}
\end{array}\right.
$$

so that

$$
\boldsymbol{P}_{\ell} \boldsymbol{P}_{h}=\boldsymbol{P}_{h} \boldsymbol{P}_{\ell}=\boldsymbol{P}_{\ell}, \ell<h
$$

and

$$
\boldsymbol{M}_{i}=\boldsymbol{X}_{i} \boldsymbol{X}_{i}^{\top}=b_{w-i} \boldsymbol{P}_{i}, i=1, \ldots, w
$$

If we consider the model

$$
\boldsymbol{y}=\sum_{i=0}^{w} \boldsymbol{X}_{i} \boldsymbol{\beta}_{i}
$$


with $\boldsymbol{\beta}_{0}$ fixed and $\boldsymbol{\beta}_{1}, \ldots \boldsymbol{\beta}_{w}$ independent with null mean vectors and variancecovariance matrices $\sigma_{i}^{2} \boldsymbol{I}_{n_{i}}, i=1, \ldots, w$, we have

$$
\boldsymbol{T}=\boldsymbol{P}_{0}
$$

Now taking

$$
\left\{\begin{array}{l}
\boldsymbol{Q}_{1}^{\circ}=\boldsymbol{P}_{1} \\
\boldsymbol{Q}_{i}^{\circ}=\boldsymbol{P}_{i}-\boldsymbol{P}_{i-1}, i=2, \ldots, w
\end{array},\right.
$$

we have mutually orthogonal orthogonal projection matrices whose sum is equal to $\boldsymbol{I}_{n}$, therefore the principal basis of a complete CJAS.

We also can see that

$$
\left\{\begin{array}{l}
\boldsymbol{M}_{1}=b_{w-1} \boldsymbol{Q}_{1}^{\circ} \\
\boldsymbol{M}_{2}=b_{w-2} \boldsymbol{Q}_{1}^{\circ}+b_{w-2} \boldsymbol{Q}_{2}^{\circ} \\
\vdots \\
\boldsymbol{M}_{w}=\boldsymbol{Q}_{1}^{\circ}+\cdots+\boldsymbol{Q}_{w}^{\circ}
\end{array}\right.
$$

so the transition matrix $\boldsymbol{B}^{\circ}$ will be invertible.

Moreover it is easy to see that we will have

$$
\left\{\begin{array}{l}
\boldsymbol{Q}_{1}=\boldsymbol{T}=\boldsymbol{P}_{0} \\
\boldsymbol{Q}_{2}=\boldsymbol{P}_{1}-\boldsymbol{P}_{0} \\
\boldsymbol{Q}_{i}=\boldsymbol{Q}_{i-1}^{\circ}=\boldsymbol{P}_{i-1}-\boldsymbol{P}_{i-2}, i=3, \ldots, w+1
\end{array}\right.
$$

as well as

$$
\left\{\begin{array}{l}
\boldsymbol{M}_{1}=b_{w-1} \boldsymbol{Q}_{1}+b_{w-1} \boldsymbol{Q}_{2} \\
\boldsymbol{M}_{2}=b_{w-2} \boldsymbol{Q}_{1}+b_{w-2} \boldsymbol{Q}_{2}+b_{w-2} \boldsymbol{Q}_{3} \\
\vdots \\
\boldsymbol{M}_{w}=\boldsymbol{Q}_{1}+\boldsymbol{Q}_{2}+\cdots+\boldsymbol{Q}_{w+1}
\end{array}\right.
$$

which enables us to write

$$
\boldsymbol{B}=\left[\begin{array}{cl}
b_{w-1} & \vdots \\
\vdots & \vdots \\
1 & \vdots
\end{array}\right]
$$




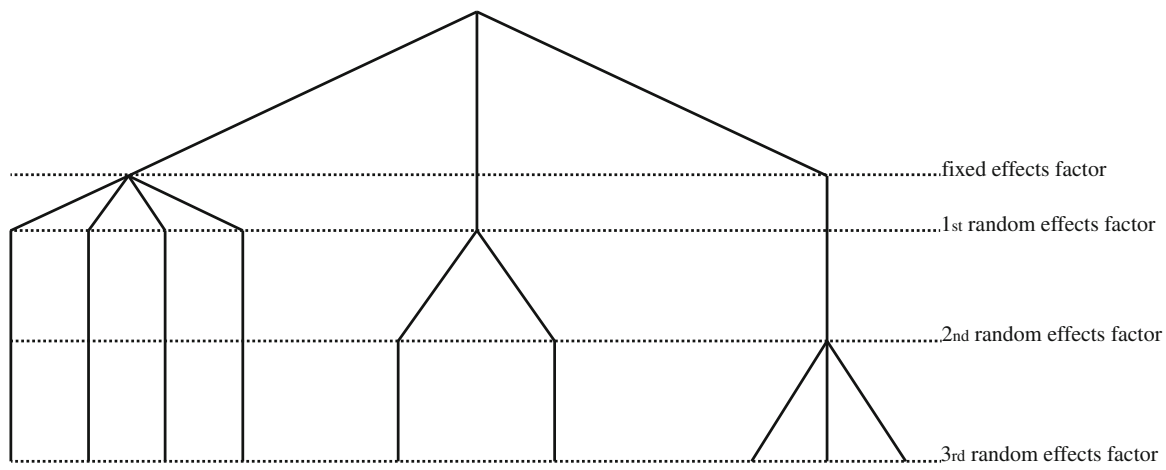

Fig. 1 Design with step nesting

As stated before, it is easy to see that the model is P-COBS, since $z^{\circ}=0$.

\subsection{Model with step nesting}

The second unbalanced model has step nesting. We keep the first factor with $a$ levels and fixed effects and replace the second one by $a$ factors with random effects that nest. But, instead of balanced nesting, we consider step nesting. Then the $r_{1}$ observations we have for the first level of the first factor will correspond to distinct levels of the first random effects factor, each nesting a single level of the remaining random effect factors. The $r_{2}$ observations for the second level of the fixed effects factor will correspond to the same level of the first random effects factor and to distinct levels of the second random effects factors and so on. This branching is presented for $a=3$ in Fig. 1 , with $r_{1}=4, r_{2}=2$ and $r_{3}=3$.

The $i$-th random effects factor will have

$$
c_{i}=\sum_{h=1}^{i} r_{h}+a-i, \quad i=1, \ldots, a
$$

levels and the model may be written as

$$
\boldsymbol{y}=\sum_{i=0}^{a} \boldsymbol{X}_{i} \boldsymbol{\beta}_{i}
$$

with

$$
\boldsymbol{X}_{0}=\boldsymbol{D}\left(\mathbf{1}_{r_{1}} \ldots \mathbf{1}_{r_{a}}\right)
$$

and

$$
\boldsymbol{X}_{i}=\boldsymbol{D}\left(\boldsymbol{X}_{i, 1} \ldots \boldsymbol{X}_{i, a}\right), i=1, \ldots, a,
$$


where

$$
\left\{\begin{array}{ll}
\boldsymbol{X}_{i, \ell}=\boldsymbol{I}_{r_{\ell},}, & \ell \leq i \\
\boldsymbol{X}_{i, \ell}=\mathbf{1}_{r_{\ell}}, & \ell>i
\end{array} .\right.
$$

We now have the

$$
\boldsymbol{Q}_{i}=\boldsymbol{D}\left(\boldsymbol{Q}_{i, 1} \ldots \boldsymbol{Q}_{i, a}\right), \quad i=1, \ldots, 2 a,
$$

with

$$
\begin{cases}\boldsymbol{Q}_{i, \ell}=\boldsymbol{Q}_{i+a, \ell}=\mathbf{0}_{r_{\ell} \times r_{\ell}}, & \ell \neq i, i=1, \ldots, a \\ \boldsymbol{Q}_{i, i}=\frac{1}{r_{i}} \boldsymbol{J}_{r_{i}}, & i=1, \ldots, a \\ \boldsymbol{Q}_{i+a, i}=\boldsymbol{K}_{r_{i}}, & i=1, \ldots, a\end{cases}
$$

where $\boldsymbol{K}_{r_{i}}=\boldsymbol{I}_{r_{i}}-\frac{1}{r_{i}} \boldsymbol{J}_{r_{i}}, i=1, \ldots, a$, and

$$
\boldsymbol{M}_{i}=\boldsymbol{X}_{i} \boldsymbol{X}_{i}^{\top}=\sum_{\ell=1}^{i}\left(\boldsymbol{Q}_{\ell}+\boldsymbol{Q}_{\ell+a}\right)+\sum_{\ell=i+1}^{a} r_{\ell} \boldsymbol{Q}_{\ell}, \quad i=1, \ldots, a
$$

so

$$
\boldsymbol{B}=\left[\begin{array}{ccccccccc}
1 & r_{2} & \ldots & r_{a} & \vdots & 1 & 0 & \ldots & 0 \\
1 & 1 & \ldots & r_{a} & \vdots & 1 & 1 & \ldots & 0 \\
\vdots & \vdots & & \vdots & \vdots & \vdots & \vdots & & \vdots \\
1 & 1 & \ldots & 1 & \vdots & 1 & 1 & \ldots & 1
\end{array}\right]
$$

In this model $w=z=a, m=2 a$ and $\boldsymbol{B}(2)$ is invertible. We thus have an unbiased S-COBS and, when normality is assumed, the estimators for variance components, either usual or canonical, will be $\operatorname{UMVUE}(\boldsymbol{Z})$.

\subsection{Model with cross-nesting}

The third model has cross-nesting with three factors. The first two are in the first model, while the third one has random effects, $c$ levels and crosses with the first one. The model may be written as

$$
\boldsymbol{y}=\sum_{i=0}^{3} \boldsymbol{X}_{i} \boldsymbol{\beta}_{i}
$$


with $\boldsymbol{\beta}_{0}$ fixed and $\boldsymbol{\beta}_{1}, \boldsymbol{\beta}_{2}$ and $\boldsymbol{\beta}_{3}$ independent, with null mean vectors and variancecovariance matrix $\sigma_{1}^{2} \boldsymbol{I}_{n^{\circ}}, \sigma_{2}^{2} \boldsymbol{I}_{c}$ and $\sigma_{3}^{2} \boldsymbol{I}_{c n^{\circ}}$, where $n^{\circ}=\sum_{j=1}^{a} r_{j}$. The model matrices will be

$$
\left\{\begin{array}{l}
\boldsymbol{X}_{0}=\boldsymbol{D}\left(\mathbf{1}_{r_{1}} \ldots \mathbf{1}_{r_{a}}\right) \otimes \mathbf{1}_{c} \\
\boldsymbol{X}_{1}=\boldsymbol{I}_{n^{\circ}} \otimes \mathbf{1}_{c} \\
\boldsymbol{X}_{2}=\boldsymbol{D}\left(\mathbf{1}_{r_{1}} \ldots \mathbf{1}_{r_{a}}\right) \otimes \boldsymbol{I}_{c} \\
\boldsymbol{X}_{3}=\boldsymbol{I}_{n^{\circ}} \otimes \boldsymbol{I}_{c}
\end{array}\right.
$$

while the mutually orthogonal orthogonal projection matrices will be

$$
\begin{cases}\boldsymbol{Q}_{j}=\boldsymbol{D}\left(\boldsymbol{Q}_{j, 1} \ldots \boldsymbol{Q}_{j, a}\right) \otimes \frac{1}{c} \boldsymbol{J}_{c}, & j=1, \ldots, a \\ \boldsymbol{Q}_{j+a}=\boldsymbol{D}\left(\boldsymbol{Q}_{j+a, 1} \ldots \boldsymbol{Q}_{j+a, a}\right) \otimes \frac{1}{c} \boldsymbol{J}_{c}, & j=1, \ldots, a \\ \boldsymbol{Q}_{j+2 a}=\boldsymbol{D}\left(\boldsymbol{Q}_{j, 1} \ldots \boldsymbol{Q}_{j, a}\right) \otimes \boldsymbol{K}_{c}, & j=1, \ldots, a \\ \boldsymbol{Q}_{j+3 a}=\boldsymbol{D}\left(\boldsymbol{Q}_{j+a, 1} \ldots \boldsymbol{Q}_{j+a, a}\right) \otimes \boldsymbol{K}_{c}, & j=1, \ldots, a\end{cases}
$$

where the sub-matrices are the

$$
\begin{cases}\boldsymbol{Q}_{j, j}=\frac{1}{r_{j}} \boldsymbol{J}_{r_{j}}, & j=1, \ldots, a \\ \boldsymbol{Q}_{j+a, j}=\boldsymbol{K}_{r_{j}}, & j=1, \ldots, a \\ \boldsymbol{Q}_{j, \ell}=\boldsymbol{Q}_{j+a, \ell}=\mathbf{0}_{r_{\ell} \times r_{\ell}}, & j \neq \ell\end{cases}
$$

with $\boldsymbol{K}_{r_{j}}=\boldsymbol{I}_{r_{j}}-\frac{1}{r_{j}} \boldsymbol{J}_{r_{j}}, j=1, \ldots, a$.

We now have

$$
\left\{\begin{array}{l}
\boldsymbol{T}=\sum_{j=1}^{a} \boldsymbol{Q}_{j} \\
\boldsymbol{M}_{1}=c \sum_{j=1}^{2 a} \boldsymbol{Q}_{j} \\
\boldsymbol{M}_{2}=\sum_{j=1}^{a} r_{j} \boldsymbol{Q}_{j}+\sum_{j=2 a+1}^{3 a} r_{j} \boldsymbol{Q}_{j} \\
\boldsymbol{M}_{3}=\sum_{j=1}^{4 a} \boldsymbol{Q}_{j}
\end{array}\right.
$$

Clearly the model is COBS with $z=a$ and

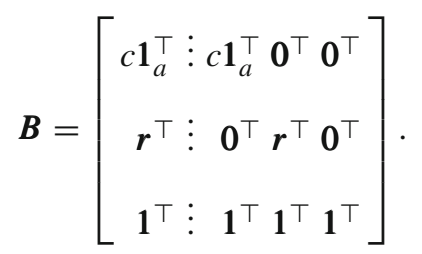

No column of $\boldsymbol{B}(1)$ is equal to any column of $\boldsymbol{B}(2)$ so the model is not P-COBS, but the row vectors of $\boldsymbol{B}(2)$ are linearly independent. Then this model is a S-COBS 
which is not P-COBS. Moreover it is unbalanced, with $w=3, z=a$ and $m=4 a$, so that $w<m-z$ since we must have $a>1$.

\subsection{Nesting into sub-models}

The fourth model also has a first fixed effects factors with $a$ levels for which we take $r_{1}, \ldots, r_{a}$ observations that will constitute the sub-models

$$
\boldsymbol{y}_{j}=\mathbf{1}_{r_{j}} \beta_{0, j}+\sum_{i=1}^{w} d_{i, j} \boldsymbol{\beta}_{i, j}, \quad j=1, \ldots, a,
$$

where the $\beta_{0, j}$ are fixed and the $\boldsymbol{\beta}_{1, j}, \ldots, \boldsymbol{\beta}_{w, j}$ are independent with null mean vectors and variance-covariance matrices $\sigma_{1}^{2} \boldsymbol{I}_{r_{j}}, \ldots, \sigma_{w}^{2} \boldsymbol{I}_{r_{j}}, j=1, \ldots, a$. We assume all these $\boldsymbol{\beta}_{i, j}, i=1, \ldots, w, j=1, \ldots, a$, to be independent, so the $\boldsymbol{y}_{1}, \ldots, \boldsymbol{y}_{a}$ will be independent with mean vectors $\mathbf{1}_{r_{1}} \beta_{0,1}, \ldots, \mathbf{1}_{r_{a}} \beta_{0, a}$ and variance-covariance matrices $\gamma_{1}^{\circ} \boldsymbol{I}_{r_{1}}, \ldots, \gamma_{a}^{\circ} \boldsymbol{I}_{r_{a}}$ with

$$
\gamma_{j}^{\circ}=\sum_{i=1}^{w} d_{i, j}^{2} \sigma_{i}^{2}=\sum_{i=1}^{w} b_{i, j}^{\circ} \sigma_{i}^{2}, j=1, \ldots, a,
$$

with $b_{i, j}^{\circ}=d_{i, j}^{2}, i=1, \ldots, w, j=1, \ldots, a$.

Then

$$
\boldsymbol{y}=\left[\begin{array}{c}
\boldsymbol{y}_{1} \\
\vdots \\
\boldsymbol{y}_{a}
\end{array}\right]=\sum_{i=0}^{w} \boldsymbol{X}_{i} \boldsymbol{\beta}_{i}
$$

where $\boldsymbol{\beta}_{0}$, with components $\beta_{0,1}, \ldots, \beta_{0, a}$, is fixed and the $\boldsymbol{\beta}_{1}, \ldots, \boldsymbol{\beta}_{a}$ are independent with null mean vectors and variance-covariance matrices $\sigma_{1}^{2} \boldsymbol{I}_{n}, \ldots, \sigma_{a}^{2} \boldsymbol{I}_{n}$. Thus $\boldsymbol{y}$ will have mean vector $\boldsymbol{X}_{0} \boldsymbol{\beta}_{0}$ with $\boldsymbol{X}_{0}=\boldsymbol{D}\left(\mathbf{1}_{r_{1}} \ldots \mathbf{1}_{r_{a}}\right)$ and variance-covariance matrix

$$
\boldsymbol{V}=\sum_{j=1}^{a} \gamma_{j}^{\circ} \boldsymbol{Q}_{j}^{\circ}
$$

with

$$
\boldsymbol{Q}_{j}^{\circ}=\boldsymbol{D}\left(\boldsymbol{Q}_{j, 1}^{\circ} \ldots \boldsymbol{Q}_{j, a}^{\circ}\right), \quad j=1, \ldots, a,
$$

where

$$
\left\{\begin{array}{ll}
\boldsymbol{Q}_{j, j}^{\circ}=\boldsymbol{I}_{r_{j}}, & j=1, \ldots, a \\
\boldsymbol{Q}_{j, j^{\prime}}^{\circ}=\mathbf{0}_{r_{j^{\prime}} \times r_{j^{\prime}}}, & j \neq j^{\prime}
\end{array} .\right.
$$


We have

$$
\boldsymbol{T}=\boldsymbol{D}\left(\frac{1}{r_{1}} \boldsymbol{J}_{r_{1}} \ldots \frac{1}{r_{a}} \boldsymbol{J}_{r_{a}}\right) \quad ; \boldsymbol{T}^{c}=\boldsymbol{D}\left(\boldsymbol{K}_{r_{1}} \ldots \boldsymbol{K}_{r_{a}}\right)
$$

and so we get

$$
\left\{\begin{array}{l}
\boldsymbol{Q}_{j}=\boldsymbol{Q}_{j}^{\circ} \boldsymbol{T}=\boldsymbol{D}\left(\boldsymbol{Q}_{j, 1} \ldots \boldsymbol{Q}_{j, a}\right) \\
\boldsymbol{Q}_{j+a}=\boldsymbol{Q}_{j}^{\circ} \boldsymbol{T}^{c}=\boldsymbol{D}\left(\boldsymbol{Q}_{j+a, 1} \ldots \boldsymbol{Q}_{j+a, a}\right)
\end{array}\right.
$$

with

$$
\begin{cases}\boldsymbol{Q}_{j, j^{\prime}}=\boldsymbol{Q}_{j+a, j^{\prime}}=\mathbf{0}_{r_{j^{\prime}} \times r_{j^{\prime}}}, & j \neq j^{\prime} \\ \boldsymbol{Q}_{j, j}=\frac{1}{r_{j}} \boldsymbol{Q}_{j}, & j=1, \ldots, a \\ \boldsymbol{Q}_{j+a, j}=\boldsymbol{K}_{r_{j}}, & j=1, \ldots, a,\end{cases}
$$

where $\boldsymbol{K}_{r_{j}}=\boldsymbol{I}_{r_{j}}-\frac{1}{r_{j}} \boldsymbol{J}_{r_{j}}, j=1, \ldots, a$, thus

$$
\left\{\begin{array}{ll}
Q_{j}^{\circ}=Q_{j}+Q_{j+a}, & j=1, \ldots, a \\
\boldsymbol{\gamma}_{j}^{\circ}=\boldsymbol{\gamma}_{j}=\boldsymbol{\gamma}_{j+a}, & j=1, \ldots, a
\end{array} .\right.
$$

Therefore we have "perfect" pairing given that

$$
\boldsymbol{B}(1)=\boldsymbol{B}(2)=\boldsymbol{B}^{\circ}
$$

since $z=a, m=2 a$ and

$$
\boldsymbol{M}_{i}=\sum_{j=1}^{a} b_{i, j}^{\circ} \boldsymbol{Q}_{j}^{\circ}=\sum_{j=1}^{a} b_{i, j}^{\circ}\left(\boldsymbol{Q}_{j}+\boldsymbol{Q}_{j+a}\right)=\sum_{j=1}^{2 a} b_{i, j} \boldsymbol{Q}_{j}, i=1, \ldots, w
$$

with

$$
b_{i, j}=b_{i, j+a}=b_{i, j}^{\circ}, i=1, \ldots, w, j=1, \ldots, a .
$$

We now have the block-wise diagonal mutually orthogonal orthogonal projection matrices

$$
\begin{cases}\boldsymbol{Q}_{j}=\boldsymbol{D}\left(\boldsymbol{Q}_{j, 1} \ldots \boldsymbol{Q}_{j, a}\right), & j=1, \ldots, a \\ \boldsymbol{Q}_{j+a}=\boldsymbol{D}\left(\boldsymbol{Q}_{j+a, 1} \ldots \boldsymbol{Q}_{j+a, a}\right), & j=1, \ldots, a\end{cases}
$$


with $\boldsymbol{Q}_{j, j}=\frac{1}{r_{j}} \boldsymbol{J}_{r_{j}}, \boldsymbol{Q}_{j+a, j}=\boldsymbol{K}_{r_{j}}, \boldsymbol{Q}_{j, \ell}=\boldsymbol{Q}_{j+a, \ell}=\mathbf{0}_{r_{\ell} \times r_{\ell}}, \ell \neq j, j=1, \ldots, a$. It is easy to see that

$$
\left\{\begin{array}{l}
\boldsymbol{T}=\sum_{j=1}^{a} \boldsymbol{Q}_{j} \\
\boldsymbol{M}_{1}=\sum_{j=1}^{2 a} \boldsymbol{Q}_{j} \\
\boldsymbol{V}=\sum_{j=1}^{2 a} \sigma^{2} \boldsymbol{Q}_{j}
\end{array}\right.
$$

Since $\boldsymbol{T}$ commutes with the $\boldsymbol{Q}_{1}, \ldots, \boldsymbol{Q}_{2 a}$, the model is COBS with

$$
\boldsymbol{B}(1)=\boldsymbol{B}(2)=\mathbf{1}_{a}^{\top}
$$

so that it is P-COBS. Moreover, since the $r_{1}, \ldots, r_{a}$ may differ, the model is not balanced.

\section{Structured families}

The mixed models with COBS

$$
\boldsymbol{y}(\ell)=\sum_{i=0}^{w} \boldsymbol{X}_{i} \boldsymbol{\beta}_{i}(\ell), \ell=1, \ldots, \bar{\ell}
$$

where vectors $\boldsymbol{\beta}_{0}(\ell), \ell=1, \ldots, \bar{\ell}$ are fixed and the $\boldsymbol{\beta}_{i}(\ell), i=1, \ldots, w, \ell=1, \ldots, \bar{\ell}$, are random, independent with null mean vectors and variance-covariance matrices $\sigma_{i}^{2} \boldsymbol{I}_{c_{i}}, i=1, \ldots, w$, are isomorphic. These models will have the same matrices $\boldsymbol{T}$ and $\boldsymbol{M}_{i}, i=1, \ldots, w$, so they will correspond to the same CJAS, $\mathscr{A}$. With

$$
\boldsymbol{Q}_{j}=\boldsymbol{A}_{j}^{\top} \boldsymbol{A}_{j}, j=1, \ldots, m,
$$

the matrices in $p b(\mathscr{A})$, the isomorphic models will have mean vectors $\boldsymbol{\mu}(\ell)=$ $\boldsymbol{X}_{0} \boldsymbol{\beta}_{0}(\ell), \ell=1, \ldots, \bar{\ell}$ and the canonical estimable vectors

$$
\boldsymbol{\eta}_{j}(\ell)=\boldsymbol{A}_{j} \boldsymbol{\mu}(\ell), \ell=1, \ldots, \bar{\ell}, j=1, \ldots, z
$$

Moreover all or none of the models in a family of isomorphic models are P-COBS. If this happens we have $z^{\circ}=0$ for all the models in the family.

For the $\eta_{j}(\ell), \ell=1, \ldots, \bar{\ell}, j=1, \ldots, z$, we have the unbiased estimators

$$
\widetilde{\boldsymbol{\eta}}_{j}(\ell)=\boldsymbol{A}_{j} \boldsymbol{y}(\ell), \ell=1, \ldots, \bar{\ell}, \quad j=1, \ldots, z
$$


Let us take

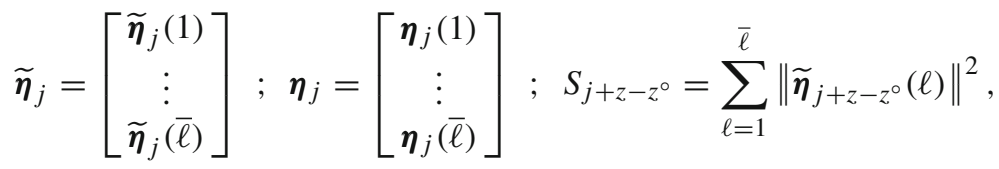

$$
\begin{aligned}
& j=z^{\circ}+1, \ldots, z \text {. }
\end{aligned}
$$

Since the $\boldsymbol{y}(\ell), \ell=1, \ldots, \bar{\ell}$, are independent, $\widetilde{\boldsymbol{\eta}}_{j}$ will be normal with mean vector $\boldsymbol{\eta}_{j}$ and variance-covariance matrix $\gamma_{j} \boldsymbol{I}_{\bar{\ell} g_{j}}$, independent of $S_{j+z-z^{\circ}}$, which is the product by $\gamma_{j}=\gamma_{j+z-z^{\circ}}$ of a central chi-square with $\bar{\ell} g_{j+z-z^{\circ}}$ degrees of freedom, $\gamma_{j} \chi_{\bar{\ell} g_{j+z-z^{\circ}}}^{2}, j=z^{\circ}+1, \ldots, z$. We could use pivot variables, in the same way as before, to carry out inference on the $\gamma_{j}, j=z+1, \ldots, m$, or on the $\eta_{j}, j=1, \ldots, z$, but we are more interested in structured families. In these families, the models are isomorphic and correspond to the treatments of a base design. The main objective will be to study the action of the factors in the base design as linear combinations $\boldsymbol{c}^{\top} \widetilde{\boldsymbol{\eta}}_{j}(\ell)$, $\ell=1, \ldots, \bar{\ell}$, of components of canonical estimable vectors, $j=1, \ldots, z$. This study is specially interesting when the base design has an orthogonal structure. Then there is an orthogonal partition

$$
\mathbb{R}^{\bar{\ell}}=\underset{d=1}{\bar{d}} \bar{\omega}_{d}
$$

where $\boxplus$ denotes the orthogonal direct sum of subspaces, and, if $\boldsymbol{\mu}^{\circ}$ is the mean vector for the base model, the relevant hypotheses for that model, are

$$
H_{0, d}: \mu^{\circ} \in \omega_{d}=\bar{\omega}_{d}^{\perp}, \quad d=1, \ldots, \bar{d}
$$

Let the row vectors of $\boldsymbol{G}_{d}$ constitute an orthonormal basis for $\bar{\omega}_{d}, d=1, \ldots, \bar{d}$. Given a normal vector $\boldsymbol{z}$ with mean vector $\boldsymbol{\mu}^{\circ}$ and variance-covariance matrix $\lambda \boldsymbol{I}_{\bar{\ell}}$ independent from $S^{\circ}$, which is the product by $\lambda$ of a central chi-square with $g^{\circ}$ degrees of freedom, $\lambda \chi_{g^{\circ}}^{2}$, the statistics

$$
\mathscr{F}_{d}=\frac{g^{\circ}}{g_{d}^{\circ}} \frac{\left\|\boldsymbol{G}_{d} \boldsymbol{z}\right\|^{2}}{S^{\circ}}, \quad d=1, \ldots, \bar{d},
$$

have $F$ distributions with $g_{d}^{\circ}=\operatorname{dim}\left(\bar{\omega}_{d}\right)$ and $g^{\circ}$ degrees of freedom and non-centrality parameters

$$
\delta_{d}=\frac{1}{\lambda}\left\|\boldsymbol{G}_{d} \boldsymbol{\mu}^{\circ}\right\|^{2}, d=1, \ldots, \bar{d},
$$

$F\left(\cdot \mid g_{d}^{\circ}, g^{\circ}, \delta_{d}\right)$, which are null when and only when the corresponding hypothesis $H_{0, d}, d=1, \ldots, \bar{d}$, hold. 
To apply these results to structured families we consider the

$$
\boldsymbol{z}_{j}(\boldsymbol{c})=\left[\begin{array}{c}
\boldsymbol{c}^{\top} \widetilde{\boldsymbol{\eta}}_{j}(1) \\
\vdots \\
\boldsymbol{c}^{\top} \widetilde{\boldsymbol{\eta}}_{j}(\bar{\ell})
\end{array}\right] ; \boldsymbol{\mu}_{j}^{\circ}(\boldsymbol{c})=\left[\begin{array}{c}
\boldsymbol{c}^{\top} \boldsymbol{\eta}_{j}(1) \\
\vdots \\
\boldsymbol{c}^{\top} \boldsymbol{\eta}_{j}(\bar{\ell})
\end{array}\right] ; j=z^{\circ}+1, \ldots, z
$$

and test the hypotheses

$$
H_{0, j, d}(\boldsymbol{c}): \boldsymbol{\mu}_{\boldsymbol{j}}^{\circ}(\boldsymbol{c}) \in \omega_{d}, j=z^{\circ}+1, \ldots, z, \quad d=1, \ldots, \bar{d}
$$

using the statistics

$$
\mathscr{F}_{j, d}(\boldsymbol{c})=\frac{\bar{l} g_{j+z-z^{\circ}}}{g_{d}^{\circ}} \frac{\left\|\boldsymbol{G}_{d} \boldsymbol{z}_{j}(\boldsymbol{c})\right\|^{2}}{S_{j+z-z^{\circ}}}, j=z^{\circ}+1, \ldots, z, \quad d=1, \ldots, \bar{d} .
$$

For instance, if in the base design we have $u$ factors, with $a_{1}, \ldots, a_{u}$ levels, that cross we will have $\bar{\ell}=\prod_{i=1}^{u} a_{i}$ treatments. We now have to test the hypothesis of absence of effects or of interactions for the factor or factors of the base design. Given

$$
\mathscr{C} \subseteq \overline{\bar{u}}=\{1, \ldots, u\}
$$

$H_{0}(\mathscr{C})$ will be the hypothesis of absence of effects or of interactions for the factor or factors with indexes in $\mathscr{C}$. Then, see Fonseca et al. (2003), we have

$$
\begin{cases}\boldsymbol{G}_{j}(\mathscr{C})=\frac{1}{\sqrt{a_{j}}} \mathbf{1}_{a_{j}}^{\top}, & j \notin \mathscr{C}, j=1, \ldots, u \\ \boldsymbol{G}_{j}(\mathscr{C})=\boldsymbol{L}_{a_{j}}, & j \in \mathscr{C}, j=1, \ldots, u\end{cases}
$$

and

$$
\begin{cases}g_{j}(\mathscr{C})=1, & j \notin \mathscr{C}, j=1, \ldots, u \\ g_{j}(\mathscr{C})=a_{j}-1, & j \in \mathscr{C}, j=1, \ldots, u\end{cases}
$$

$\nabla$

If the model in the family were the two factor models we have been considering we could consider hypothesis on

$$
\boldsymbol{\mu}_{1}^{\circ}=\left[\begin{array}{c}
\beta_{0, \bullet}(1) \\
\vdots \\
\beta_{0, \bullet}(\bar{\ell})
\end{array}\right]
$$


this is on the vector of mean values for the components of $\boldsymbol{\beta}_{0}(1), \ldots, \boldsymbol{\beta}_{0}(\bar{\ell})$, or on

$$
\boldsymbol{\mu}_{2}^{\circ}(\boldsymbol{c})=\left[\begin{array}{c}
\boldsymbol{c}^{\top} \boldsymbol{L}_{a} \boldsymbol{\beta}_{0}(1) \\
\vdots \\
\boldsymbol{c}^{\top} \boldsymbol{L}_{a} \boldsymbol{\beta}_{0}(\bar{\ell})
\end{array}\right]=\left[\begin{array}{c}
\left(\boldsymbol{L}_{a}^{\top} \boldsymbol{c}\right)^{\top} \boldsymbol{\beta}_{0}(1) \\
\vdots \\
\left(\boldsymbol{L}_{a}^{\top} \boldsymbol{c}\right)^{\top} \boldsymbol{\beta}_{0}(\bar{\ell})
\end{array}\right] .
$$

It is interesting to point out that, whatever $\boldsymbol{c}$, the sum of components of $\boldsymbol{L}_{a}^{\top} \boldsymbol{c}$ is null. Thus the components of $\boldsymbol{\mu}_{2}^{\circ}(\boldsymbol{c})$ will be contrasts on the components of the $\boldsymbol{\beta}_{0}(1), \ldots, \boldsymbol{\beta}_{0}(\bar{\ell})$.

The previous discussion shows clearly the interplay of two different structures:

- that of the models in the family;

- that of the base design

in the joint analysis of structured families of models.

\section{Final remarks}

The use of CJAS in the study of COBS led to:

1. the definition of statistics $\widetilde{\boldsymbol{\eta}}_{j}, j=1, \ldots, z$ and $S_{j}, j=z+1, \ldots, m$, which are relevant in estimating the canonical estimable vectors, $\boldsymbol{\eta}_{j}, j=1, \ldots, z$ and the canonical variance components $\gamma_{j}, j=z+1, \ldots, m$. These statistics play, once normality is assumed, an important part in defining pivot variables and, thus, carrying out inference;

2. special types of models, S-COBS and P-COBS for which we get additional results on the estimation of variance components, both usual and canonical. Moreover for P-COBS we also have enhanced use of pivot variables in testing hypotheses on estimable vectors.

Besides isolated models, structured families were considered. It is interesting to point out that the treatments in the base model can correspond to different sets of experimental conditions. Then the joint analysis of the models in the family enables the study of the action of those experimental conditions.

All these developments were illustrated using a two factor model that was considered throughout our discussion.

The four cases presented in Sect. 4, clearly shows the wide range of possible applications. Furthermore, the example that was used along most part of the paper, improves the assessment of this approach, giving explicit formulae useful for computational purposes.

As an example, for instance, we could consider an experiment with grapevines planted in rows. The first factor would be the distance between grapevines and the second the clones. We recall that grapevines having vegetative reproduction can be grouped into castes, having a mythical ancestor. Grapevine castes are divided into a 
large number of clones whose ancestors are known. The clones to be considered would belong to some well know caste.

Acknowledgments The authors would like to thank the anonymous referees for useful comments and suggestions. This work was partially supported by CMA / FCT / UNL, under the project PEstOE/MAT/UI0297/2014, and by the Center of Mathematics, University of Beira Interior under the project PEst-OE/MAT/UI0212/2014.

\section{References}

Caliński T, Kageyama S (2000) Block designs. A randomization approach. Volume I: analysis. Lecture notes in statistics. Springer, Berlin

Caliński T, Kageyama S (2003) Block designs. A randomization approach. Volume II: design. Lecture notes in statistics. Springer, Berlin

Carvalho F, Mexia JT, Oliveira MM (2008) Canonic inference and commutative orthogonal block structure. Discuss Math Prob Stat 28(2):171-181

Fonseca M, Mexia JT, Zmyślony R (2003) Estimators and tests for variance components in cross nested orthogonal models. Discuss Math Probab Stat 23:173-201

Fonseca M, Mexia JT, Zmyślony R (2006) Binary operation on Jordan algebras and orthogonal normal models. Linear Algebr Appl 417(1):75-86

Fonseca M, Mexia JT, Zmyślony R (2007) Jordan algebras, generating pivot variables and orthogonal normal models. J Interdiscip Math 10(2):305-326

Fonseca M, Mexia JT, Zmyślony R (2008) Inference in normal models with commutative orthogonal block structure. Acta et Commentationes Universitatis Tartunesis de Mathematica 12:3-16

Jordan P, von Neumann J, Wigner E (1934) On the algebraic generalization of the quantum mechanical formalism. Ann Math 35(1):29-64

Kariya T, Kurata H (2004) Generalized least squares. Wiley, New York

Khuri AI, Mathew T, Sinha BK (1998) Statistical tests for mixed linear models. Wiley series in probability and statistics. Wiley, New York

Lehmann EL, Casella G (2003) Theory of point estimation, 2nd edn. Springer Texts in Statistics

Mejza S (1992) On some aspects of general balance in designed experiments. Statistica 52(2):263-278

Mexia JT (1987) Multi-treatment regression designs. Trabalhos de Investigação, No 1. Departamento de Matemática, Faculdade de Ciências e Tecnologia, Universidade Nova de Lisboa

Mexia JT (1990) Best linear unbiased estimates, duality of $F$ tests and the Scheff multiple comparison method in presence of controlled heterocedasticity. Comput Stat Data Anal 10:271-281

Michalski A, Zmyślony R (1996) Testing hypothesis for variance components in mixed linear models. Statistics 27(3-4):297-310

Moreira EE, Ribeiro AB, Mateus E, Mexia JT, Ottosen LM(2005a) Regressional modelling of electrodialytic removal of $\mathrm{Cu}, \mathrm{Cr}$ and As from CCA timber waste: application to sawdust. Wood Sci Technol 39(4):291309

Moreira EE, Mexia JT, Ribeiro AB, Mateus E, Ottosen LM (2005b) Regressional modelling of electrodialytic removal of $\mathrm{Cu}, \mathrm{Cr}$ and $\mathrm{As}$ from $\mathrm{CCA}$ timber waste: application to wood chips. Listy Biometryczne 42(1):11-23

Moreira E, Mexia JT (2007) Multiple regression models with cross nested orthogonal base model. In: Proceedings of the 56th session of the ISI 2007-International Statistical Institute, Lisboa

Moreira E (2008) Família estruturada de modelos com base ortogonal: teoria e aplicações, Ph.D. Thesis, Faculdade de Ciências e Tecnologia, Universidade Nova de Lisboa (in Portuguese)

Nelder JA (1965a) The analysis of randomized experiments with orthogonal block structure. I. Block structure and the null analysis of variance. Proc R Soc Lond Ser A Math Phys Sci 283(1393):147-162

Nelder JA (1965b) The analysis of randomized experiments with orthogonal block structure. II. Treatment, structure and the general analysis of variance. Proc R Soc Lond Ser A Math Phys Sci 283(1393):163-178

Schott JR (1997) Matrix analysis for statistics. Wiley series in probability and statistics. Wiley, New York Seely J (1970a) Linear spaces and unbiased estimators. Ann Math Stat 41(5):1725-1734

Seely J (1970b) Linear spaces and unbiased estimators. Application to a mixed linear model. Ann Math Stat 41(5):1735-1748 
Seely J (1971) Quadratic subspaces and completeness. Ann Math Stat 42(2):710-721

Seely J, Zyskind G (1971) Linear spaces and minimum variance unbiased estimation. Ann Math Stat 42(2):691-703

Scheffé H (1959) The analysis of variance, Wiley series in probability and statistics. Wiley, New York

VanLeeuwen DM, Seely JF, Birkes DS (1998) Sufficient conditions for orthogonal designs in mixed linear models. J Stat Plan Inference 73(1-2):373-389

VanLeeuwen DM, Birkes DS, Seely JF (1999) Balance and orthogonality in designs for mixed classification models. Ann Stat 27(6):1927-1947

Zmyślony R (1980) A characterization of Best Linear Unbiased Estimators in the general linear model. Lecture Notes in Statistics, vol 2. Springer, pp 365-373

Zmyślony R, Drygas H (1992) Jordan algebras and bayesian quadratic estimation of variance components. Linear Algebra Appl 168:259-275 